\title{
A nucleotide-switch mechanism mediates opposing catalytic activities of Rel enzymes
}

\author{
Hedvig Tamman ${ }^{1,10}$, Katleen Van Nerom ${ }^{1,10}$, Hiraku Takada ${ }^{2,3}$, Niels Vandenberk ${ }^{4}$, Daniel Scholl ${ }^{5}$,

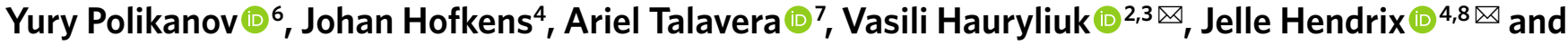 \\ Abel Garcia-Pino ${ }^{1,9}$ 压
}

Bifunctional Rel stringent factors, the most abundant class of RelA/SpoT homologs, are ribosome-associated enzymes that transfer a pyrophosphate from ATP onto the $3^{\prime}$ of guanosine tri-/diphosphate (GTP/GDP) to synthesize the bacterial alarmone (p)ppGpp, and also catalyze the $3^{\prime}$ pyrophosphate hydrolysis to degrade it. The regulation of the opposing activities of Rel enzymes is a complex allosteric mechanism that remains an active research topic despite decades of research. We show that a guanine-nucleotide-switch mechanism controls catalysis by Thermus thermophilus $\operatorname{Rel}\left(\operatorname{Rel}_{\mathrm{Tt}}\right)$. The binding of GDP/ATP opens the N-terminal catalytic domains (NTD) of $\operatorname{Rel}_{T t}\left(\operatorname{Rel}_{T t}{ }^{\mathrm{NTD}}\right.$ ) by stretching apart the two catalytic domains. This activates the synthetase domain and allosterically blocks hydrolysis. Conversely, binding of ppGpp to the hydrolase domain closes the NTD, burying the synthetase active site and precluding the binding of synthesis precursors. This allosteric mechanism is an activity switch that safeguards against futile cycles of alarmone synthesis and degradation.

T he stringent response enables bacteria to drastically reduce RNA synthesis among a myriad of metabolic readjustments that result in a strong phenotypic resetting ${ }^{1}$. This response allows bacteria, including major pathogens, to cope with adverse environmental changes and is required for stress survival and virulence. Originally dubbed 'the magic spots ${ }^{2}$, alarmone nucleotides guanosine tetra- and penta-phosphate (collectively referred to as (p)ppGpp) are the linchpin and effector molecules of the stringent response that orchestrate the phenotypic switch from rapid to slow growth ${ }^{3}$.

The cellular level of (p)ppGpp ${ }^{2,3}$ is controlled by the action of RelA/SpoT homolog (RSH) enzymes ${ }^{1,4,5}$. The paradigm of RSH catalysis is based on the structure of Streptococcus dysgalactiae Rel NTD $\left(\operatorname{Rel}_{s e q}{ }_{\text {NTD }}\right)$ solved more than a decade ago ${ }^{6}$. $\operatorname{Rel}_{\text {seq }}{ }^{\mathrm{NTD}}$ is a truncated variant of $\mathrm{Rel}_{\text {seq }}$ that includes only the two catalytic domains with opposing activities - ppGpp hydrolase (HD) and ppGpp synthetase $(\mathrm{SYN})^{6}$-and lacks the C-terminal regulatory domains of the enzyme involved in binding of the ribosome and recognition of deacylated transfer RNA (see Supplementary Fig. 1a that shows the domain composition of a typical 'long' RSH enzyme ${ }^{4}$ ). The two $\operatorname{Rel}_{\text {seq }}{ }_{\text {NTD }}$ molecules observed in the same crystal lattice were locked in contrasting conformations leading to the hypothesis of reciprocal regulation of SYN and HD domains. However, the enzyme contained a nucleotide bound in both active sites in one of the conformations and only one active site occupied in the other one. This was a surprising finding, considering earlier observations indicated catalysis was incompatible with the simultaneous activation of synthetase and hydrolase function ${ }^{7,8}$ and underlined the complexity of the enzyme conformational landscape. Therefore, to further under- stand how nucleotides regulate the opposite reactions catalyzed by Rel, it is essential to solve the structures of Rel with catalytically engaged SYN or HD domains.

Here, we determined the structures of $\operatorname{Rel}_{T t}^{\mathrm{NTD}}$ in a resting unbound state and in different states compatible with active catalysis. These active states are consistent with a postcatalytic active synthetase state (open) and an active hydrolase state (closed). The open state contains AMP and ppGpp bound in the SYN domain, whereas the closed state has ppGpp bound in the HD domain. This structural work together with a detailed single-molecule Förster resonance energy transfer (smFRET) study of the effect of nucleotides on the conformational ensemble of the enzyme reveals that a guanine-nucleotide-allosteric switch mechanism controls the bifunctional catalysis of T. thermophilus $\operatorname{Rel}\left(\operatorname{Rel}_{T t}\right)$.

The activation of one catalytic domain involves the stabilization of a conformational state incompatible with the opposing catalytic activity. In this way, the binding of GDP to the SYN domain triggers an open form of $\operatorname{Re}_{T t}{ }_{T T}^{\mathrm{ND}}$ that aligns the catalytic residues of the synthetase domain and switches off the hydrolase activity. Conversely, binding of ppGpp in the catalytic site of the hydrolase domain triggers the closing of $\operatorname{Rel}_{T t}^{\mathrm{NTD}}$, thus precluding the binding of substrates to the SYN domain. These structural features underpin the contribution of the allosteric control by nucleotide substrates that effectively switches the enzymatic activity to prevent futile catalytic cycles.

\section{Results}

Structure of nucleotide-free $\operatorname{Rel}_{T t}^{\mathrm{NTD}}$ in a resting state. The remarkable structural plasticity of Rel has limited the determination of high-resolution structures of the enzyme from different bacterial

\footnotetext{
'Cellular and Molecular Microbiology, Faculté des Sciences, Université Libre de Bruxelles, Brussels, Belgium. ²Department of Molecular Biology, Umeå University, Umeå, Sweden. ${ }^{3}$ Laboratory for Molecular Infection Medicine Sweden, Umeå University, Umeå, Sweden. ${ }^{4}$ Molecular Imaging and Photonics, Chemistry Department, KU Leuven, Leuven, Belgium. ${ }^{5}$ SFMB, Université Libre de Bruxelles, Brussels, Belgium. ${ }^{6}$ Department of Biological Sciences, College of Liberal Arts and Sciences, University of Illinois at Chicago, Chicago, IL, USA. ${ }^{7}$ CMMI, Université Libre de Bruxelles, Gosselies, Belgium. ${ }^{8}$ Dynamic Bioimaging Laboratory, Advanced Optical Microscopy Centre and Biomedical Research Institute, Hasselt University, Agoralaan C (BIOMED), Hasselt, Belgium. ${ }^{9} \mathrm{WELBIO}$, Brussels, Belgium. ${ }^{10}$ These authors contributed equally: Hedvig Tamman, Katleen Van Nerom. ${ }^{凶}$ e-mail: vasili.hauryliuk@umu.se; jelle. hendrix@uhasselt.be; agarciap@ulb.ac.be
} 
species for years. Therefore, we used T. thermophilus Rel as experimental system ${ }^{9}$, given that the expected high thermodynamic stability of this enzyme would be an advantage for structural studies. We constructed a truncated version of the enzyme ${ }^{9}$ containing only the two catalytic domains ( $\operatorname{Rel}_{T t}{ }_{T}^{\mathrm{NTD}}$, amino acid positions $1-355$, designed in the same manner as the $\operatorname{Rel}_{S e q}{ }^{\text {NTD }}$ truncated variants ${ }^{6}$ ) to directly assess the effect of nucleotide binding in the proposed allosteric mechanism that controls the bifunctional catalysis typical of Rel.

The structure of nucleotide-free $\operatorname{Rel}_{T t}{ }^{\text {NTD }}$ (Fig. 1a and Supplementary Fig. 1b-e) recapitulates the structures of $\operatorname{Rel}_{S e q}{ }^{\text {NTD }}$ (ref. ${ }^{6}$ ), Mycobacterium tuberculosis Rel $\mathrm{NTD}^{10}\left(\right.$ RelA $_{M t b}{ }^{\mathrm{NTD}}$ ) as well as the mono-functional synthetase-only Escherichia coli RSH RelA ${ }^{11}$ $\left(\mathrm{RelA}_{E c}\right)$ both in terms of the individual fold of domains and their relative orientations. Only the conformation of $\alpha$-helices $\alpha 6, \alpha 7$ and loop $\alpha 6-\alpha 7$ are noticeably different (Supplementary Fig. 1d-f). In RelA $_{E c}$ the $\alpha 6-\alpha 7$ loop is projected toward the pseudo-hydrolase site of $\mathrm{RelA}_{E c}$, effectively blocking the site, whereas in $\operatorname{Rel}_{T t}{ }^{\mathrm{NTD}}$, $\operatorname{RelA}_{M t b}{ }^{\mathrm{NTD}}$ and $\operatorname{Rel}_{S e q}{ }^{\mathrm{NTD}} \alpha 6-\alpha 7$ is partially disordered and pointing in an opposite direction ${ }^{6,11-13}$ (Supplementary Fig. 1g,h).

Nucleotide-free $\operatorname{Rel}_{T t}{ }^{\mathrm{NTD}}$ is observed in two different conformations in the same crystal lattice, with both conformations displaying a similar spatial arrangement of domains. The main difference between the two conformations is that the $\alpha 6-\alpha 7$ motif of the hydrolase domain is projected away from the active site, and the $\alpha 6 \alpha$-helix is largely unwound to an extended conformation (Supplementary Fig. 1h). Although this extreme conformation is primarily stabilized by the lattice contacts and likely a result of the crystallization process, it underscores the highly dynamic nature of the hydrolase domain, in particular the $\alpha 6-\alpha 7$ motif and its importance for catalysis.

Structure of $\operatorname{Rel}_{\mathrm{Tt}}{ }^{\mathrm{NTD}}$ bound to ppGpp. $\operatorname{Rel}_{T t}{ }^{\mathrm{NTD}}$ hydrolase activity is virtually undetectable at $4^{\circ} \mathrm{C}$ (Supplementary Fig. 2a and Supplementary Table 1), which is not surprising given that the optimal growth temperature of T. thermophilus is about $65^{\circ} \mathrm{C}$. The low enzymatic activity enabled cocrystallization in the presence of the native ppGpp substrate.

From the structure of the $\operatorname{Rel}_{T t}{ }^{\mathrm{NTD}}$-ppGpp complex (Fig. 1b and Supplementary Fig. 2b), it is immediately apparent that the complex is in a more compacted conformation than the resting state of $\operatorname{Rel}_{T t}{ }^{\mathrm{NTD}}$, diverging from all other known conformations of Rel and RelA enzymes (Fig. 1a,b). In the complex, ppGpp makes a large number of contacts with the enzyme (Fig. 1c) and is bound in a conformation reminiscent of that of $\mathrm{ppG2} 2^{\prime}: 3^{\prime} \mathrm{p}$ (a GDP-2': $3^{\prime}$-cyclic monophosphate derivative of the ppGpp hydrolysis) observed in the active site of $\operatorname{Rel}_{\text {Seq }}{ }^{\text {NTD }}$ (Supplementary Fig. 3a) and nicotinamide adenine dinucleotide phosphate (NADP) bound to a single-domain human small alarmone hydrolase (SAH) RSH Mesh1 (ref. ${ }^{14}$ ) (Protein Data Bank (PDB) ID 5VXA) (Supplementary Fig. 3b). The guanine base of ppGpp is stacked between R43, R44 and M157 and makes hydrogen bonds with S45, N150 and T153, while the ribose makes a van der Waals interaction with $\mathrm{N} 150$ and a hydrogen bond with Y49. The $2^{\prime}$ and $3^{\prime}$ oxygen atoms from the ribose are held very close (within $4.5 \AA$ ) to the $\mathrm{Mn}^{2+}$ ion and N150. In addition, the $\mathrm{Mn}^{2+}$ ion is $2.5 \AA$ apart from an oxygen atom of the $3^{\prime}$ phosphate of ppGpp. This strong coordination confirms the essential role of the metal ion in the activation of the phosphorus center for a nucleophilic attack (Fig. 1c).

The hydrolase active site has a remarkable distribution of surface electrostatics. The site consists of a deep and wide cavity with one half of the site positively charged and involved in the stabilization of the $5^{\prime}$-polyphosphate groups of the substrate and the other predominately acidic and more directly involved in the $3^{\prime}$-pyrophosphate hydrolysis (Supplementary Fig. 3c). The 3'-pyrophosphate group of ppGpp is bound in nearly the same position as that of $\mathrm{ppG}^{\prime}: 3^{\prime} \mathrm{p}$
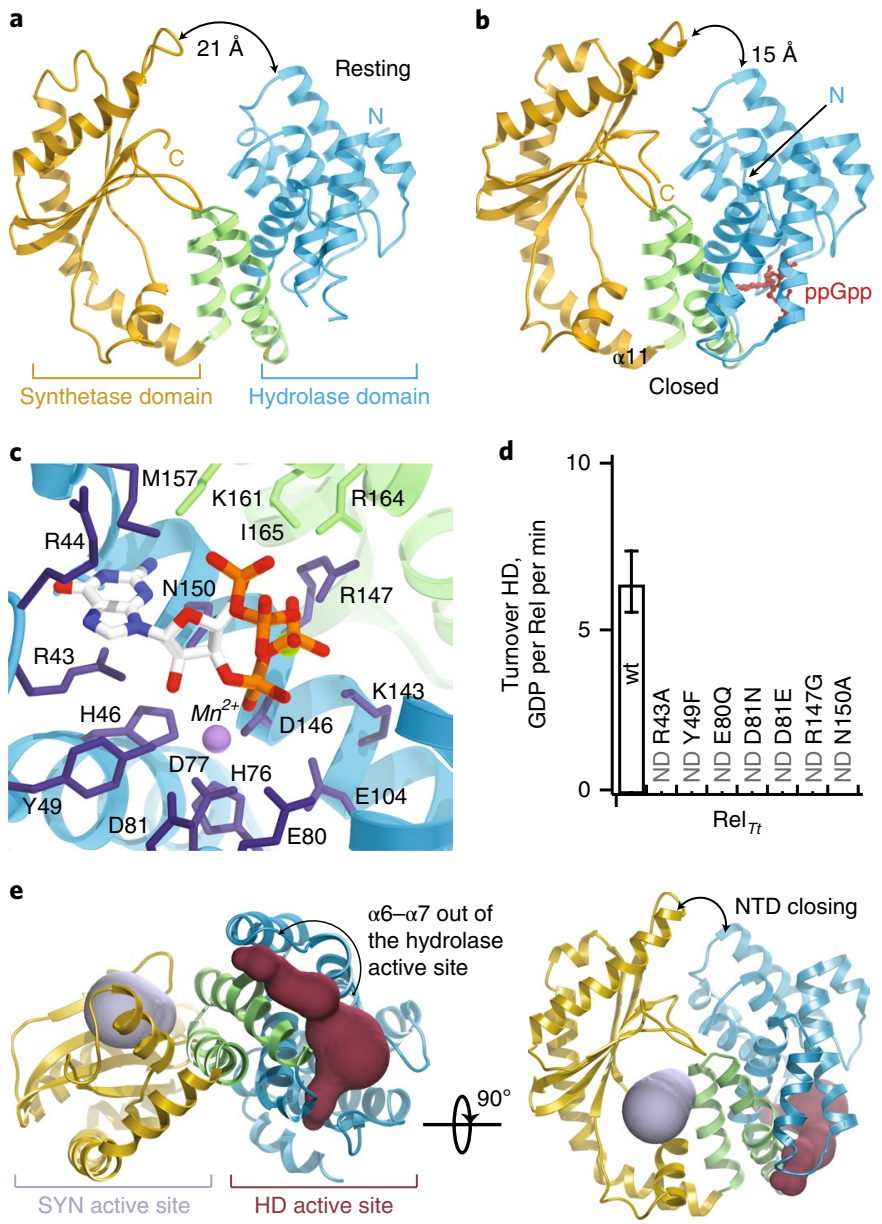

Fig. 1 Structure of $\operatorname{Rel}_{T t}{ }^{\text {NTD }}$ in a resting and closed state. a, Structure of $\mathrm{Rel}_{T t}^{\text {NTD }}$ in the resting, nucleotide-free state. The HD domain is colored light blue, the $\alpha 9-\alpha 10 \alpha$-helical substructure connecting the HD and the SYN domains is colored light green and the SYN domain is colored orange. b, Structure of Rel ${ }_{T t}^{N T D}$ in the active hydrolase state (closed state, colored as in a), bound to ppGpp (shown in red). Interdomain distances were estimated in every case from the $\mathrm{N}$ atom of the $\mathrm{Y} 18$ to the $\mathrm{N}$ atom of T287. c, Details of the Rel $_{T t}{ }^{N T D}-p p G p p$ binding interface, all important catalytic residues as well as the $\mathrm{Mn}^{2+}$ ion are labeled in the figure. $\mathbf{d}$, Impact of active site substitutions on the hydrolase activity of $\operatorname{ReI}_{T t}$, based on the interactions observed in the crystal structure of the $\operatorname{Rel}_{T t}{ }_{T T}{ }_{T}$-ppGpp complex. Substitutions affecting the direct interaction with the guanine base of ppGpp (R43A), the ribose (Y49F), residues involved in catalysis (D81N, D81E and N150A) or interfering with the accommodation of the phosphate groups of ( $p$ )ppGpp (R147G) universally abrogate hydrolysis. Turnovers were estimated by linear regression of the individual kinetic series. ND, not determined; wt, wild type. e, HD and SYN active sites (shown in red and light blue volumes, respectively) in the closed state as observed in the $\operatorname{Re}_{T t}{ }_{T T}{ }^{N T}$-ppGpp complex. The allosteric arrangements involved in the active site setup are coupled to the closing of the enzyme that constricts the synthetase active site and prevents ppGpp synthesis.

(Supplementary Fig. 3a) in the acidic half of the active site formed by D77, E80, D81, E104 and D146, which is crucial for catalysis. This acidic section of the active site has already been highlighted as a functional hot $\operatorname{spot}^{6}$. Substitutions in the conserved $\mathrm{HDX}_{3} \mathrm{ED}$ motif $^{15}$ (either $\mathrm{HDX}_{3} G \mathrm{G}$ or $\mathrm{HDX}_{3} \mathrm{EV}$ ) completely abrogate hydrolysis in $\operatorname{Rel}_{\text {Seq }}$ NTD (ref. ${ }^{6}$ ). The motif diverged to ${ }_{82} \mathrm{FPLADA}_{87}$ in the inactive hydrolase domain of $E$. coli RelA $_{E c}$ indicating this could be one of the factors that contributes to the lack of activity of the specialized E. coli synthetase. Indeed, conservative substitutions of 
E80 (E80Q) and D81 (D81N) or even changes in the distance of the carboxylate group relative to ppGpp in the HD domain (as in D81E) have a strong effect on the activity of $\operatorname{Rel}_{T t}$ (Fig. 1d).

The $5^{\prime}$-pyrophosphate group of ppGpp is stabilized by the damping effect of K112, K143, R147 and K161 and projects toward the $\alpha$-helix $\alpha 6$ (Fig. 1c). From this binding mode, we propose that the relative spatial arrangement between $\alpha 6$ and $\alpha 9$ would determine the specificity of the hydrolase function. Indeed, as observed in the $\operatorname{Rel}_{\text {Seq }}{ }^{\mathrm{NTD}}-\mathrm{ppG} 2^{\prime}: 3^{\prime} \mathrm{p}$ and hMesh1-NADP complexes, it is the local disposition of $\alpha 6$ and $\alpha 9$ that allows accommodation of the 5 '-pyrophosphate and nicotinamide riboside groups (Supplementary Fig. 3a,b). As with the case of the acidic patch, substitutions at this positive section (R147G) also strongly affect hydrolysis (Fig. 1d).

HD-domain priming involves occlusion of the SYN domain. A crucial observation from the $\operatorname{Rel}_{T t}{ }^{\mathrm{NTD}}$-ppGpp complex is that the entire SYN domain moves $6 \AA$ closer to the HD domain compared to the resting state, sterically occluding and switching off the synthetase active site (Fig. 1a,b,e and Supplementary Fig. 4). In addition, the $\alpha 6-\alpha 7$ motif of the HD domain becomes fully structured and moves about $85^{\circ}$ away from the position occupied by $\alpha 6-\alpha 7$ in the inactive HD domain of RelA $A_{E c}$. This movement of $\alpha 6-\alpha 7$ aligns together all the residues that constitute the aforementioned positive patch and allows the binding of ppGpp in the active site. As a result, the hydrolase site of the enzyme becomes fully accessible.

This contrasts with the synthetase domain that becomes even more confined in the active hydrolase state (Fig. 1e and Supplementary Fig. 4). The ATP binding site of the SYN domain is particularly affected. Residues R249, H252, and R277 of the ATP binding pocket become buried, with $\mathrm{H} 252$ tethering the SYN active site to the linker region via stacking interactions with W187. Moreover, residues D272 and E345 directly involved in catalysis are misaligned in a conformation that is not compatible with ATP hydrolysis. This observation confirms that in the active hydrolase state the synthetase function is likely precluded.

Structure of $\operatorname{Rel}_{T t}{ }^{\mathrm{NTD}}$ in an active synthetase state. To understand how the nucleotide substrates control the synthesis of ppGpp in the SYN domain, we solved the structure of $\operatorname{Rel}_{T t}{ }_{T T}$ in a postcatalytic state (Fig. 2a) in complex with the reaction products AMP and ppG$\mathrm{p}_{\mathrm{N}} \mathrm{p}$ (Fig. $2 \mathrm{a}-\mathrm{c}$ and Supplementary Fig. 5a). We used APPNP, a $\beta-\gamma$ phosphate nonhydrolysable ATP analog, that reacts exceedingly slowly with GDP in the active center of $\operatorname{Rel}_{T t}{ }^{\mathrm{NTD}}$ yielding $\mathrm{ppGp}_{\mathrm{N}} \mathrm{p}$ (Supplementary Fig. 5b,c and Supplementary Table 1).

The structure of $\operatorname{Rel}_{T t}{ }^{\mathrm{NTD}}$ in this postcatalytic conformation (Fig. 2a) shows a contrasting picture with the $\operatorname{Rel}_{T t}{ }^{\mathrm{NTD}}$-ppGpp complex (Fig. 1b). The presence of two nucleotides in the synthetase site is accompanied by domain rearrangements that stretch both domains almost $45 \AA$ apart, resulting in the opening of the NTD (Fig. 2a). This movement disengages $\mathrm{H} 252$ from the W187 tether, which now coordinates Q213 destabilising $\alpha 11$.

The comparison of the two opposing conformations of the two active catalytic states suggest a potential route for transduction of the allosteric signal (Fig. $2 \mathrm{c}, \mathrm{d}$ ). The central $\alpha$-helix bundle motif of the $\operatorname{Rel}_{T t}{ }_{\text {NTD }}$ enzyme forms a small hydrophobic core with $\alpha 13$ of the SYN domain that connects both catalytic domains ${ }^{6}$. The wedging effect of the nucleotides is thus spread toward the HD domain via the $\alpha 13$ 'transmission' core that has swiveled orthogonally to the $\alpha 9$ dipole approximately $60^{\circ}$ (Fig. $2 \mathrm{~d}-\mathrm{f}$ ). This fractures $\alpha 11$ in two ( $\alpha 11^{\prime}$ and $\left.\alpha 11^{\prime \prime}\right)$, exposing the SYN domain active site and stretching the HD domain away. This conformational rearrangement portrays a much larger allosteric effect associated with the switch to an active synthetase state than that expected from the two conformations of $\operatorname{Rel}_{\text {Seq }}{ }^{\mathrm{NTD}}$, which involved a rotation of $10^{\circ}$, stretching the two domains approximately $2.4 \AA$ apart ${ }^{6}$. Considering the lattice constraints and the partial occupancy of nucleotides in both active
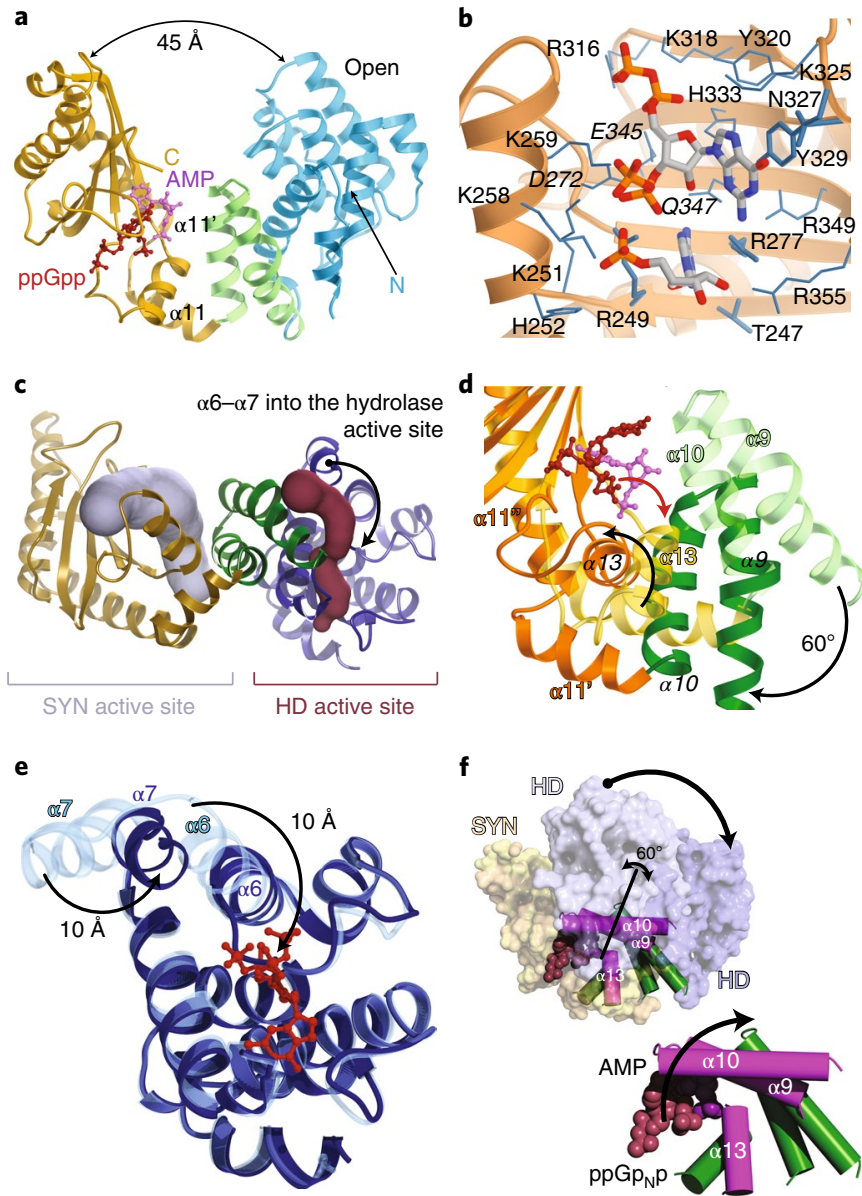

Fig. 2 | Structure of $\operatorname{Rel}_{T t}{ }^{\mathrm{NTD}}$ in the open active SYN state, molecular bases of the interdomain allosteric interplay. $\mathbf{a}$, Structure of Rel $_{T t}^{N T D}$ in the active synthetase state (open state, colored as Fig. 1a), bound to $p p G p_{N} p$ (shown in red) and AMP (shown in purple). Interdomain distances were estimated from the $\mathrm{N}$ atom of the $\mathrm{Y} 18$ to the $\mathrm{N}$ atom of T287. b, Details of the Rel ${ }_{T t}^{N T D}-$ ppGp $_{N} \mathrm{p}-\mathrm{AMP}$ binding interface, all important catalytic residues are labeled in the figure with residues directly involved in catalysis shown in bold. c, Active site representation of the enzyme in the open form $\left(\mathrm{Re}_{T t}^{\mathrm{NTD}}-\mathrm{pp} \mathrm{Gp}_{\mathrm{N}} \mathrm{p}\right.$-AMP complex) colored as in Fig. 1e. The stretching of the two catalytic domains to activate the synthetase catalytic site is coupled to the closing and inactivation of the HD catalytic site. $\mathbf{d}$, Superposition of the SYN domain of the closed (in light colors) and open (in dark colors) states illustrating the rigid body rotation movement of the $\alpha 9-\alpha 10-\alpha 13$ 'transmission core' induced by the binding of nucleotides, which results in the opening of the enzyme. e, Superposition of the HD domain of the closed (light blue) and open (dark blue) states showing the allosteric changes triggered by the activation of the SYN domain (as well as partial occlusion of HD domain) and the activation of the HD domain (relocation of the $\alpha 6-\alpha 7$ snaplock). f, Superposition of the open (in dark colors) and closed conformations (in light colors) of Rel Tt $_{T+}^{\text {NTD }}$. The $\alpha 9-\alpha 10-\alpha 13$ 'transmission core' is shown in magenta for the closed state and in green for the open state. The rigid body swivel of the transmission core triggers the opening of the enzyme and partial occlusion of the HD domain active site.

sites of $\operatorname{Rel}_{\text {Seq }}{ }^{\mathrm{NTD}}$, it is not surprising that the enzyme is observed in an intermediate state closer to the resting state than to either active catalytic conformations.

Substrate binding to the SYN domain aligns active site. The postcatalytic state of $\operatorname{Rel}_{T t}{ }^{\mathrm{NTD}}$ resembles the precatalytic state observed in the structure of the Staphylococcus aureus single-domain small 


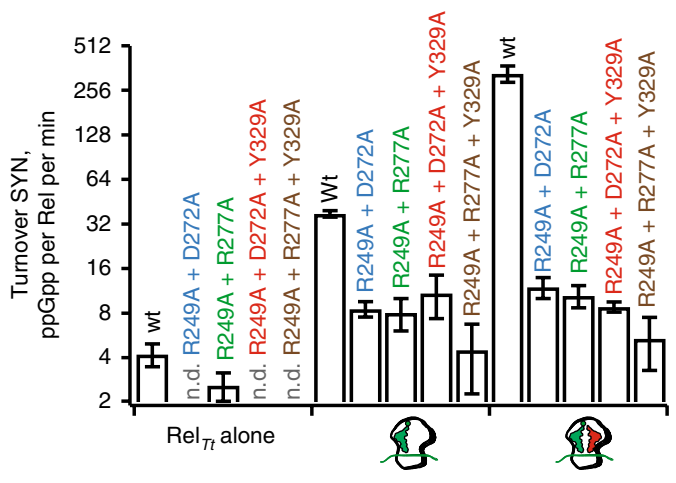

b

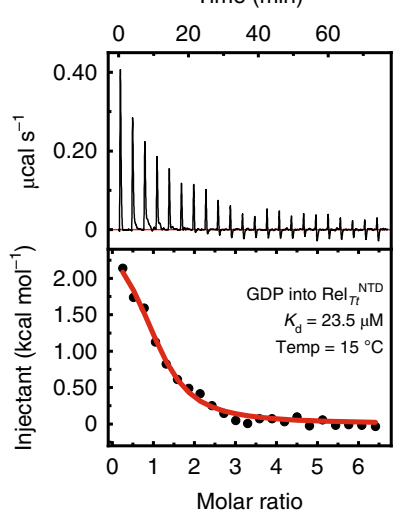

c

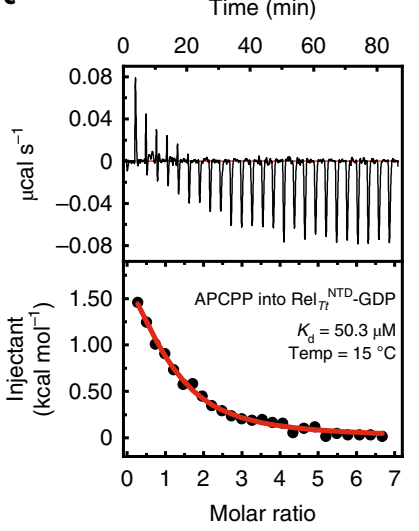

Fig. 3 | Nucleotide binding controls Rel $\mathrm{I}_{T t}$ allosteric switch, off the ribosome. a, SYN activity of $\mathrm{Re}_{T t}$ and R249A/D272A, R249A/R277A, R249A/D272A/ Y329, R249A/R277A/Y329 substituted versions alone, and activated by either T. thermophilus 70S ribosome initiation complex (70S IC) or the 70S IC with deacylated tRNA ${ }^{\mathrm{Val}}$ in the A site. In every case, ND refers to 'not determined/detectable' activity. Turnovers were estimated by linear regression of the individual kinetic series. $\mathbf{b}$, Titration of GDP into $\operatorname{Re}_{T t}^{N T D}$. $\mathbf{c}$, Titration of APCPP into Rel ${ }_{T t}^{\text {NTD }}$ in the presence of saturating amounts of GDP.

alarmone synthetase (SAS) RelP. The overall interactions of $p p G p_{N} p$ with the active site of the $\operatorname{Rel}_{T t}{ }_{T t D}$ SYN domain are similar to those observed in the RelP-GTP-adenosine- $5^{\prime}-((\alpha, \beta)$-methyleno)triphosphate (APCPP, an $\alpha-\beta$ nonhydrolysable ATP analog) (PDB ID 6EWZ) and RelP-pppGpp (PDB ID 6EX0) complexes $^{16}$ (Fig. 2a and Supplementary Fig. 6a,b). We observed only small differences in the accommodation of the ligand and the orientation of the $\beta 3-\beta 4_{319-331}$ loop, or G-loop ${ }^{17}$, that contains the conserved $\mathrm{Y}_{329}$ residue involved in coordinating the guanine base in the active site (Supplementary Fig. 1c and 6b). These differences are likely due to the lack of the additional phosphate group that is present in both RelP complexes ${ }^{16}$. The coordination of the adenosine group of AMP in the active site also resembles that of the precatalytic RelP with the adenosine base stacked between R249 and R277 and the $\alpha$-phosphate of AMP coordinated in the same manner as that observed in the RelP-GTPAPCPP complex by R249 and K215 (Supplementary Fig. 6b). In addition, the $\beta 1-\alpha 13$ loop and $\alpha 13$ contribute a patch of positive residues that stabilize the pyrophosphate group transferred to ppG$\mathrm{p}_{\mathrm{N}} \mathrm{p}$, which is around $2.0 \AA$ away from the site it would occupy in a precatalytic state, similar to that of RelP, as part of APCPP.

These two active site elements are observed in a similar orientation to that of the $\beta 1-\alpha 2$ loop and $\alpha 2$ of RelP, coordinating the triphosphate groups of APCPP (Supplementary Fig. 6b). Indeed, mutations that destabilize ATP binding and affect the interaction of GDP/GTP with the G-loop (R249A, D272A, R277A and Y329A) have a strong impact on the activity of the native full length $\operatorname{Rel}_{T t}$ when it is assayed by itself or when the enzyme is activated by either T. thermophilus $70 \mathrm{~S}$ ribosome initiation complex (70S IC) or the 70S IC with deacylated tRNA ${ }^{\mathrm{Val}}$ in the A-site, the ultimate natural activator of ppGpp synthesis by $\operatorname{Rel}_{T t}$ (Fig. 3a).

GDP opens the SYN domain to induce the active synthetase. To directly address the sequential binding hypothesis, we monitored the binding of these nucleotides using isothermal titration calorimetry (ITC). GDP binds $\operatorname{Rel}_{T t}{ }^{\mathrm{NTD}}$ with a $K_{\mathrm{d}}$ of $23 \mu \mathrm{M}$ (Fig. $3 \mathrm{~b}$ and Supplementary Table 2), and in excellent agreement with the smFRET data APCPP alone does not bind $\operatorname{Rel}_{T t}{ }_{\text {NTD }}$ (Supplementary Fig. 7a and Supplementary Table 2). However, once the $\operatorname{Rel}_{T t}{ }_{\text {NTD }}$-GDP complex is formed, APCPP binds with an affinity of around $50 \mu \mathrm{M}$ (Fig. $3 \mathrm{c}$ and Supplementary Table 2).

The binding of both GDP and APCPP is entropically driven (Supplementary Fig. 7b and Supplementary Table 2). In the case of the binding of GDP to the SYN domain, the release of ordered water molecules from the GDP binding cleft is accompanied by the movement of $\alpha 6-\alpha 7$ that partially occludes the HD active center and an increase in the enzyme flexibility as observed in the crystal structure.

These structural changes are consistent with entropically driven binding events. The ITC data suggest that the binding of GDP is coupled to the opening of the enzyme that reveals the otherwise buried ATP binding site. In addition, it creates an 'entropy reservoir' that drives the binding of APCPP with the the release of water molecules from the now exposed binding cleft. This supports the notion that off the ribosome GDP must 'open' the enzyme to reveal the ATP binding site, as predicted from the analysis of the crystallographic data. It remains to be determined as to whether such a feature is still present in the context of the enhancing effect of the ribosome.

Activation of SYN domain precludes ppGpp binding to HD. The active synthetase conformation observed in the $\mathrm{Rel}_{T t}{ }^{\mathrm{NTD}}$-AMP-ppGp $\mathrm{N}$ complex involves local allosteric rearrangements in the HD domain that are coupled to the opening of the enzyme. The $\alpha 6-\alpha 7$ loop approaches toward the catalytic residues of the hydrolase center seemingly blocking the access to the active site of the HD domain (Fig. 2f). The side chain of Y111 in the $\alpha 6-\alpha 7$ loop occupies the binding site of the guanine base of ppGpp and the rest of the $\alpha 6-\alpha 7$ loop covers the active site in a fuzzy manner typical of a local disordered motif. Indeed, these structural changes would effectively close the hydrolase site, reducing its radius to almost half its size and expanding the radius and dimensions of the synthetase active site to accommodate both nucleotide substrates (Supplementary Fig. 4).

These changes in conformation also induce a misalignment of the HD active site residues. Most of the residues directly involved in hydrolysis remain in position; however, the side chains of residues K112, K143 and K161 involved in the coordination of ppGpp become disordered or go out of position. Additionally, residues 80 to 82 from the $\alpha 4-\alpha 5$ loop move away from the active site with residue D81, in particular, completely displaced. Given the importance of the ${ }^{80} \mathrm{E}-\mathrm{D}^{81}$ motif in catalysis, the cumulative effect of all these allosteric changes likely results in a complete hydrolysis switch off.

From the structure of the free $\operatorname{Rel}_{T t}{ }^{\text {NTD }}$ and $\operatorname{Rel}_{S e q}{ }^{\text {NTD }}$ bound to GDP it is clear that only the presence of both substrates in the active site of the synthetase domain can trigger such large domain rearrangements. This is an important observation in the context of the catalytic mechanism of the enzyme. Once the Rel is no longer involved in active synthesis and returns to the resting state, the 
a
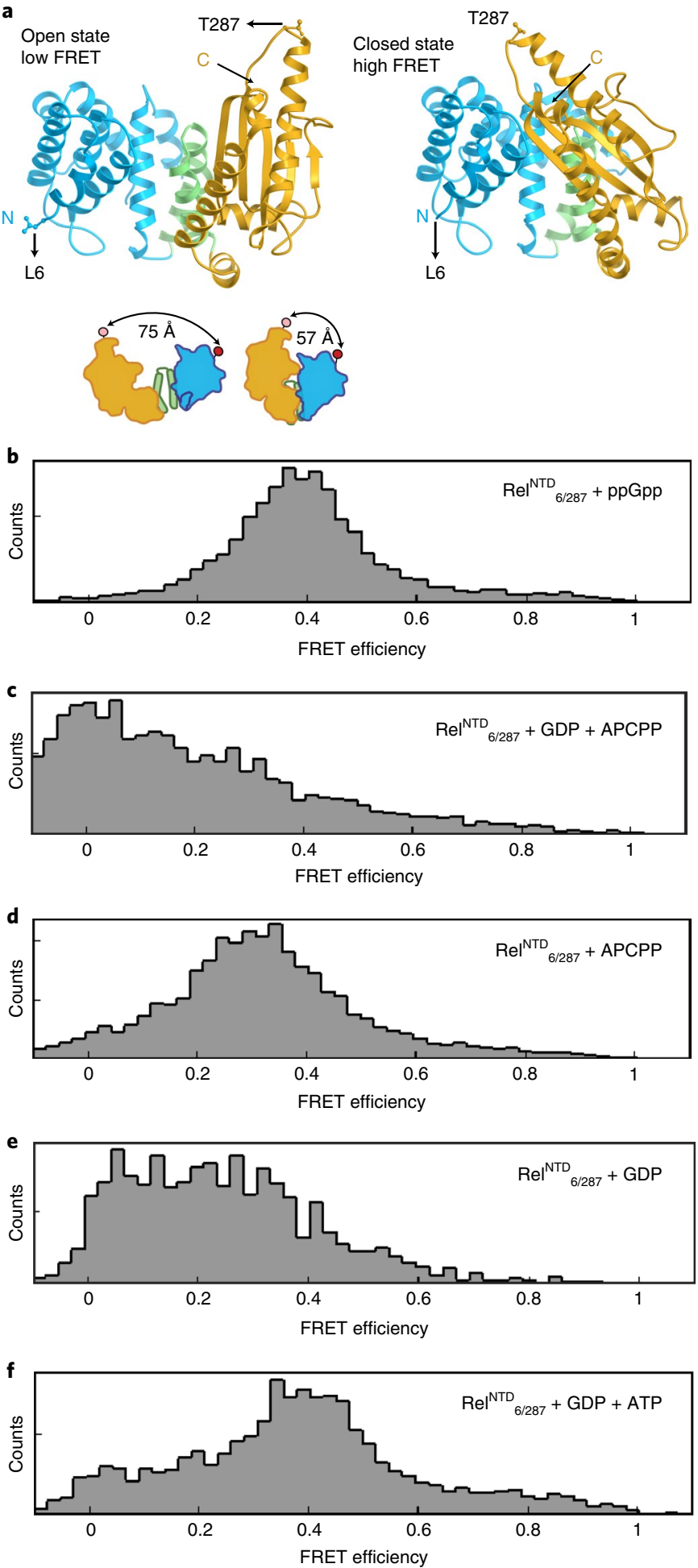

Fig. 4 | Rel ${ }_{T t}^{\text {NTD }}$ catalytic domain conformational dynamics in the presence of nucleotides assessed by smFRET. $a$, Structural models of Rel $\mathrm{I}_{T+}{ }^{\text {NTD }}$ in the open and closed conformations (to probe the interdomain movements associated with the nucleotides allosteric control). Dye attachment sites are L6C and T287C. b-f, One-dimensional histograms of the FRET efficiency of the $\operatorname{Rel}_{T t}^{\text {NTD }}{ }_{6 / 287}$ in the presence of ppGpp (b), GDP + APCPP (c), APCPP (d), GDP (e) and GDP + ATP (f). The analysis of the smFRET data suggest that the high FRET state of the enzyme in the presence of ppGpp and APCPP is consistent with the closed form observed in the crystal structure of the complex with ppGpp. By contrast, the low FRET state of the enzyme observed in the presence of GDP and GDP + APCPP is consistent with the open state of the enzyme observed in the structure of $\operatorname{Rel}_{T t}^{N T D}-p p G p_{N} p-A M P$.
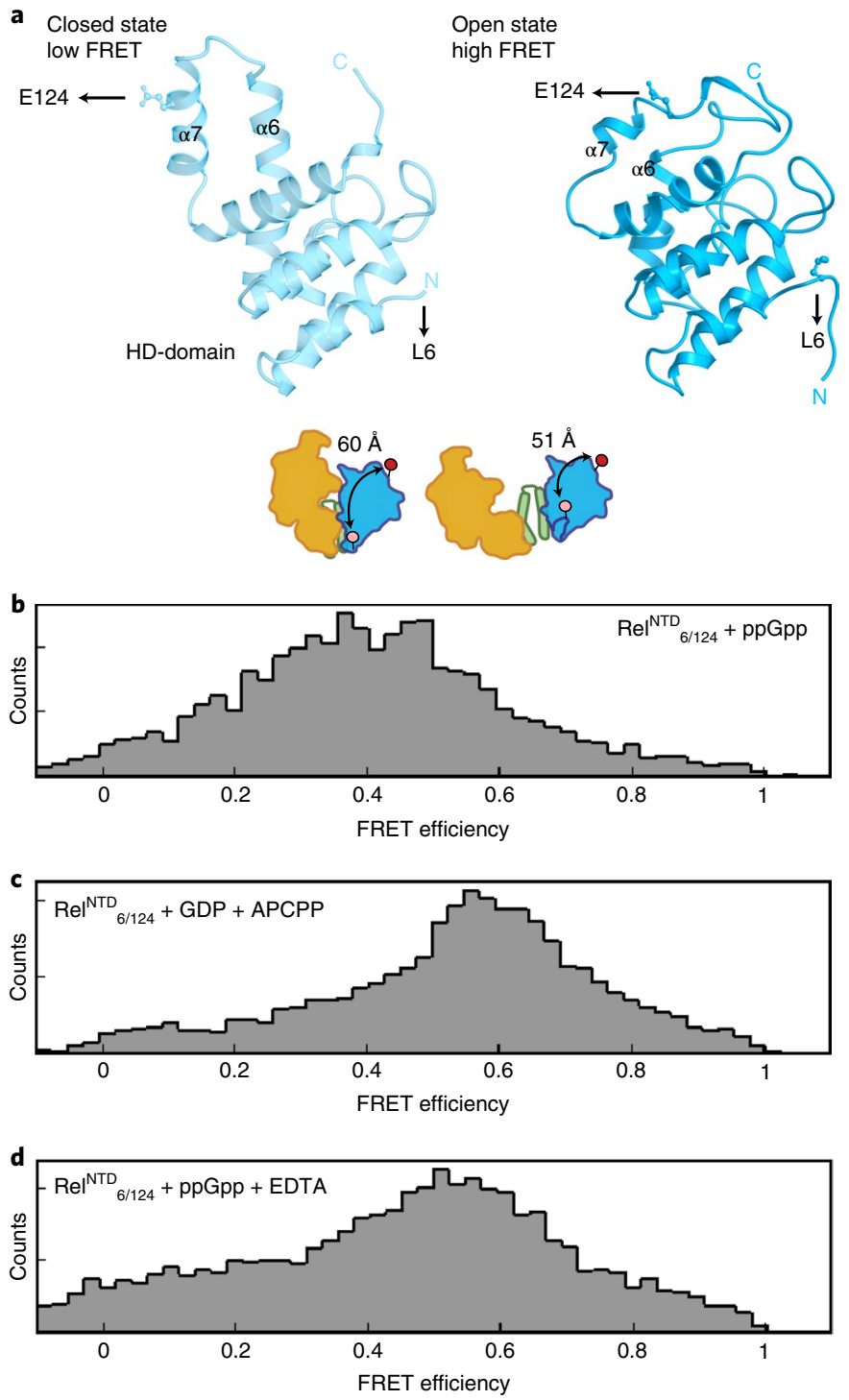

Fig. 5 | Conformational dynamics of the $\alpha 6-\alpha 7$ motif is coupled to nucleotide binding. a, Structural models of $\operatorname{Rel}_{T t}^{\mathrm{NTD}}$ in the open and closed conformations. Dye attachment sites are L6C and E124C (to probe HD-domain movements associated with the nucleotides allosteric control). b-d, One-dimensional histograms of the FRET efficiency of the Rel $\left.\right|_{T t} ^{N T D}{ }_{6 / 124}$ in the presence of ppGpp (b), GDP + APCPP (c) and ppGpp + EDTA (d). The lower FRET state of the hydrolase domain in the presence of $p p G p p$ is consistent with the movement of $\alpha$-helices $\alpha 6$ and $\alpha 7$ that allows the binding of the substrate into the active site. By contrast, the higher FRET state observed in the presence of GDP + APCPP is compatible with the snaplock switch that closes the hydrolase state coupled to the activation of the synthetase domain and the overall stretching of both catalytic domains.

$\alpha 6-\alpha 7$ loop disengages from the active site of the HD domain. This would facilitate the rapid switch to an active hydrolase conformation induced by the binding of (p)ppGpp to the HD site.

Nucleotide binding stabilizes active catalytic states. Our structural data suggest that the presence of nucleotides in either the hydrolase or the synthetase domain would prime the enzyme for that particular function, switching off the other catalytic site. Such an allosteric effect would manifest itself in the form of changes in the width of the conformational landscape that would tilt the dynamic equilibrium of the population ensemble toward the favored state 


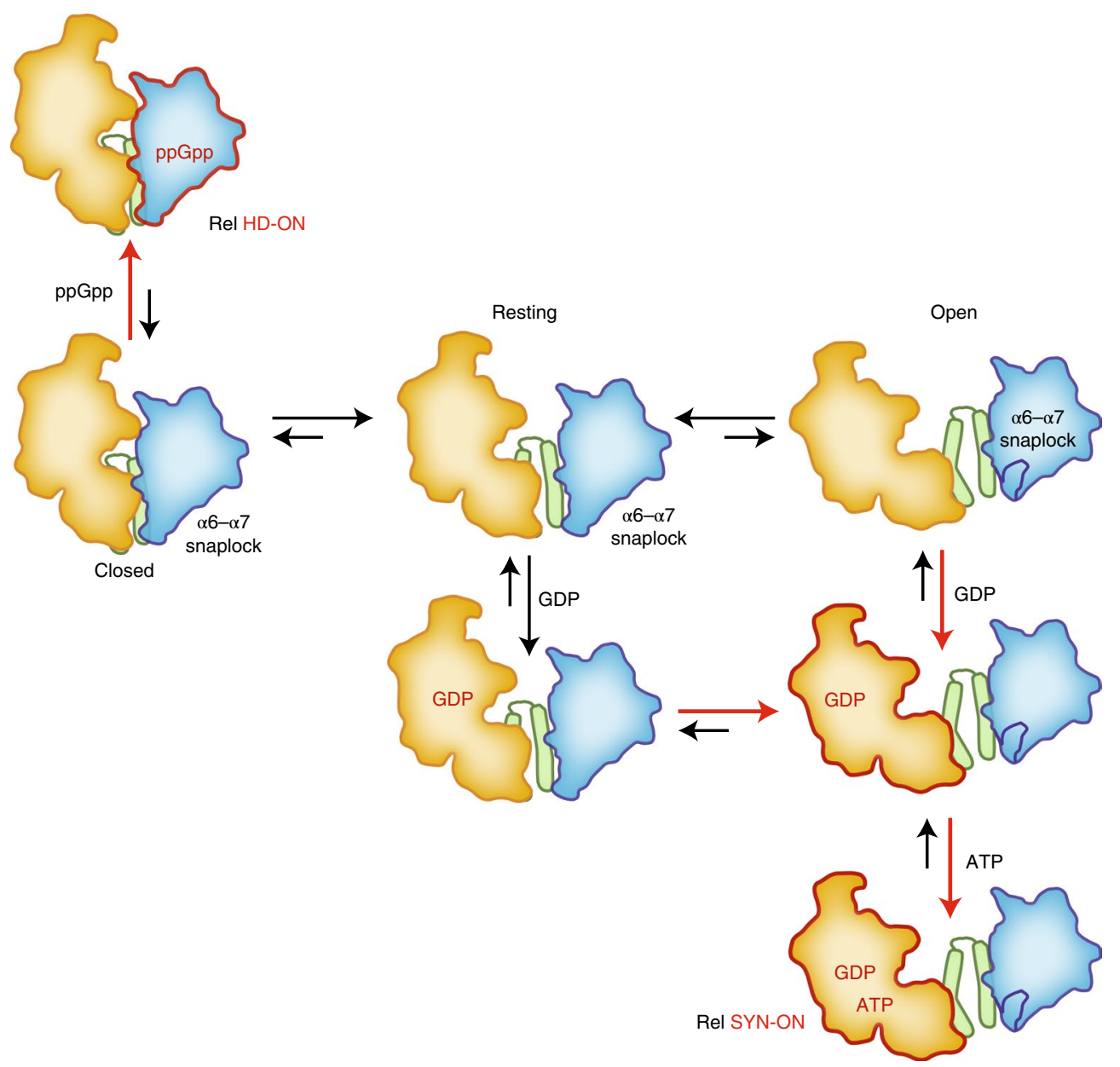

Fig. 6 | Regulation of Rel catalytic activities by substrate nucleotides. In the nucleotide-free state, $\operatorname{Re}_{T t}{ }_{T T}$ is in a resting conformation with a partially disordered HD catalytic site and the SYN domain partially occluded with the ATP binding site completely buried. In this state, neither of the catalytic domains is in a conformation compatible with active catalysis. The ppGpp synthesis cycle is initiated by GDP binding, which stabilizes the interaction of the HD domain (in blue) active site with the $\alpha 6-\alpha 7$ snaplock, and prevents ppGpp binding. This, in turn, triggers the opening of the SYN domain (in orange) active site, allowing the ATP binding and consequent synthesis of ppGpp. Therefore, the opening and activation of the SYN domain is allosterically coupled to changes in the active site of the HD domain that preclude hydrolysis. Conversely, ppGpp binding to the HD domain releases the $\alpha 6-\alpha 7$ snaplock, triggering the overall closure of the enzyme. This establishes a hydrolysis-compatible conformation with the HD active site residues correctly aligned and the active site of the SYN domain completely occluded. This conformational change safeguards against nonproductive ppGpp synthesis.

as a function of the concentration of the nucleotides in solution. We directly challenge this hypothesis using smFRET. For this, we constructed $\operatorname{Rel}_{T t}{ }^{\mathrm{NTD}}{ }_{6 / 287}$, a variant of $\operatorname{Rel}_{T t}{ }_{T \text { ND }}$ that allows fluorescent labels to be attached at cysteine residues introduced at positions 6 and 287 (Fig. 4a). The FRET-averaged interdye distance $\left\langle R_{\mathrm{DA}}\right\rangle_{\mathrm{E}}$ predicted for $\operatorname{Rel}_{T t}{ }_{T T}^{\mathrm{NTD}}{ }_{6 / 287}$ based on our crystal structures is $75 \AA$ for the open form ( $\operatorname{Rel}_{T t}{ }_{T T}$-AMP-ppGp $p_{\mathrm{N}}$ p complex) and $57 \AA$ for the closed form $\left(\operatorname{Rel}_{T t}{ }^{\mathrm{NTD}}-\mathrm{ppGp} \mathrm{p}_{\mathrm{N}} \mathrm{p}\right.$ complex).

While in the presence of ppGpp, $\operatorname{Rel}_{T t}{ }_{6 / 287}$ shows a homogenous population with $\left\langle R_{\mathrm{DA}}\right\rangle_{\mathrm{E}}$ of $61 \AA$ (Fig. $4 \mathrm{~b}$ and Supplementary Fig. 8a), when incubated with GDP combined with APCPP the $\operatorname{Rel}_{T t}{ }^{\text {NTD }}{ }_{6 / 287}$ population shifts to a $\left\langle R_{\mathrm{DA}}\right\rangle_{\mathrm{E}}$ of $70 \AA$ consistent with the opening of the enzyme (Fig. $4 \mathrm{c}$ and Supplementary Fig. $8 \mathrm{~b}$ ). These clearly different conformational states match the theoretical states expected from the aforementioned crystal structures (Figs. 1b and $2 \mathrm{a})$. In the presence of APCPP alone, $\operatorname{Rel}_{T t}{ }^{\mathrm{NTD}}{ }_{6 / 287}$ remains in the closed conformation $\left(\left\langle R_{\mathrm{DA}}\right\rangle_{\mathrm{E}}\right.$ of $61 \AA$ ) (Fig. $4 \mathrm{~d}$ and Supplementary Fig. $8 \mathrm{c})$. Conversely, GDP triggers the opening of the enzyme $\left(\left\langle R_{\mathrm{DA}}\right\rangle_{\mathrm{E}}\right.$ of $71 \AA$ ) (Fig. $4 \mathrm{e}$ and Supplementary Fig. $8 \mathrm{~d}$ ). When ATP was added after pre-incubating the enzyme with GDP, the ppGpp produced as a result of the reaction returns the equilibrium to the closed state (Fig. 4f and Supplementary Fig. 8e). This suggests the reaction occurs in a sequential manner with the guanine-nucleotide substrates binding first in the active site.

The $\alpha 6-\alpha 7$ motif is an allosteric 'snaplock' that controls HD. We considered that the motions of the $\alpha 6-\alpha 7$ loop were coupled to the allosteric switching of the enzyme, constituting a crucial element of the intramolecular crosstalk between domains. In the hydrolase-ON state ( $\operatorname{Rel}_{T t}{ }^{\text {NTD }}$-ppGpp complex, closed state), this loop was projected away from the hydrolase active site allowing the binding of ppGpp, whereas in the synthetase-ON state $\left(\mathrm{Rel}_{T t}^{\mathrm{NTD}}\right.$ postcatalytic open state) $\alpha 6-\alpha 7$ moved toward the hydrolase active site precluding the binding of ppGpp, effectively switching off the hydrolase function.

To directly probe this assumption with smFRET, we labeled $\operatorname{Rel}_{T t}{ }_{T t D}$ in positions 6 and $124\left(\operatorname{Rel}_{T t}{ }^{\text {NTD }}{ }_{6 / 124}\right)$ via cysteine residues introduced at these positions (Fig. 5a). The latter residue located in $\alpha$-helix $\alpha 7$, was used to probe the conformational change of the $\alpha 6-\alpha 7$ motif on nucleotide binding.

In the presence of ppGpp, $\operatorname{Rel}_{T t}{ }^{\text {NTD }}{ }_{6 / 124}$ is observed in a low FRET state of $\left\langle R_{\mathrm{DA}}\right\rangle_{\mathrm{E}}=61 \AA$, indicating displacement of the loop away from the active site (Fig. $5 \mathrm{~b}$ and Supplementary Fig. 8f). Conversely, 
when bound to GDP and APCPP, the enzyme switched to a high FRET state $\left(\left\langle R_{\mathrm{DA}}\right\rangle_{\mathrm{E}}=55 \AA\right)$ indicative of the loop movement toward the active site (Fig. $5 \mathrm{c}$ and Supplementary Fig. $8 \mathrm{~g}$ ). These smFRET data are in good agreement with $\left\langle R_{\mathrm{DA}}\right\rangle_{\mathrm{E}}$ estimates based on the structural data that predicts a distance between dyes of $51 \AA$ for the open state and $60 \AA$ for the closed. The removal of the $\mathrm{Mn}^{2+}$ ion from the hydrolase site by incubation with EDTA, limited the closed conformation even in the presence of ppGpp (Fig. $5 \mathrm{~d}$ and Supplementary Fig. 8h).

These observations support the role of $\alpha 6-\alpha 7$ as an allosteric 'snaplock'. When the snaplock is folded away from the hydrolase active site, the enzyme is predominantly in the closed state and in the hydrolase-ON conformation. Conversely, engagement of the snaplock leads to closure of the hydrolase active site and the 'snap'-opening of the enzyme, thus exposing the synthetase active site.

\section{Discussion}

The dynamic modulation of the cellular alarmone levels is paramount to maintenance of cellular homeostasis. Here we showed that Rel enzymes possessing active hydrolase and synthetase domains rely on additional levels of allosteric regulation besides the control that the C-terminal regulatory domains exert ${ }^{11,18,19}$, which prevents the occurrence of futile catalytic cycles. The activation of one of the catalytic domains entails the physical blockade and active site misalignment of the other. Our results support the view that the allosteric motion of $\alpha$-helices $\alpha 6$ and $\alpha 7$ is coupled to the catalytic cycle, precluding or allowing the access of substrate to the hydrolase active site (Figs. 1e, 2e and 6) whereas the relative conformational state between domains regulates the synthetase function, hindering access to the synthetase site in the closed state (Figs. 2c,d and 6). This allosteric control provides a bona fide on/off switch that renders one domain completely blocked while the other is active (Fig. 6). In addition, our results suggest that the allosteric control of long $\mathrm{RSH}$ enzymes in the absence of the ribosome may differ from that of SASs such RelP or RelQ that also bind their substrates in an ordered sequence but incorporate ATP first and then GDP or GTP ${ }^{20}$. We show that in long RSH enzymes this sequence is reversed. This divergence in the catalytic mechanism is most likely due to the loss of the hydrolase domain and with it, the requirement of triggering an open state in SASs to bind ATP.

The regulation of catalysis of bifunctional enzymes is usually dominated by allosteric transitions preventing the occurrence of futile cycles $^{21}$. The current view of the role of nucleotides in the control of Rel enzymes, based on the structure of $\mathrm{Rel}_{\text {seq }}$, does not provide a conclusive explanation as to how the conformational interplay between the two catalytic domains is linked to the activation of one site to the detriment of the other. Moreover, in all available structures of different RSH enzymes the two catalytic domains are observed in similar conformations in which the ATP binding site in the SYN domain is buried, resulting in conformations that are incompatible with an active synthetase state ${ }^{6,10-13}$

The nucleotide-switch mechanism described in this study provides the structural basis for the control of Rel enzymes by its nucleotide substrates. We show that during catalysis, when one of the enzymatic domains is active the other one is allosterically impaired. In this context, the effect of enhancers of catalysis (such as the docking platform formed by the ribosome-deacylated tRNA complex ${ }^{11-13,18,22}$ or the phospho-transferase system ${ }^{23}$ ) would trigger and stabilize conformations of the enzyme compatible with a particular catalytic state that act together with the additional allosteric regulation by nucleotides. The synchronous action of these regulatory layers constitutes a tightly regulated mechanism that prevents the occurrence of futile catalytic cycles and facilitates the effect of the alarmone as a bacterial phenotypic switch.

\section{Online content}

Any methods, additional references, Nature Research reporting summaries, source data, extended data, supplementary information, acknowledgements, peer review information; details of author contributions and competing interests; and statements of data and code availability are available at https://doi.org/10.1038/s41589020-0520-2.

Received: 22 June 2019; Accepted: 13 March 2020;

Published online: 11 May 2020

\section{References}

1. Laffler, T. \& Gallant, J. A. Stringent control of protein synthesis in E. coli. Cell 3, 47-49 (1974).

2. Cashel, M. \& Gallant, J. Two compounds implicated in the function of the RC gene of Escherichia coli. Nature 221, 838-841 (1969).

3. Hauryliuk, V., Atkinson, G. C., Murakami, K. S., Tenson, T. \& Gerdes, K. Recent functional insights into the role of (p)ppGpp in bacterial physiology. Nat. Rev. Microbiol. 13, 298-309 (2015).

4. Atkinson, G. C., Tenson, T. \& Hauryliuk, V. The RelA/SpoT homolog (RSH) superfamily: distribution and functional evolution of ppGpp synthetases and hydrolases across the tree of life. PLOS ONE 6, e23479 (2011).

5. Stent, G. S. \& Brenner, S. A genetic locus for the regulation of ribonucleic acid synthesis. Proc. Natl Acad. Sci. USA 47, 2005-2014 (1961).

6. Hogg, T., Mechold, U., Malke, H., Cashel, M. \& Hilgenfeld, R. Conformational antagonism between opposing active sites in a bifunctional RelA/SpoT homolog modulates (p)ppGpp metabolism during the stringent response [corrected]. Cell 117, 57-68 (2004).

7. Avarbock, D., Salem, J., Li, L. S., Wang, Z. M. \& Rubin, H. Cloning and characterization of a bifunctional RelA/SpoT homologue from Mycobacterium tuberculosis. Gene 233, 261-269 (1999).

8. Mechold, U., Murphy, H., Brown, L. \& Cashel, M. Intramolecular regulation of the opposing (p)ppGpp catalytic activities of Rel(Seq), the Rel/Spo enzyme from Streptococcus equisimilis. J. Bacteriol. 184, 2878-2888 (2002).

9. Van Nerom, K., Tamman, H., Takada, H., Hauryliuk, V. \& Garcia-Pino, A. The Rel stringent factor from Thermus thermophilus: crystallization and X-ray analysis. Acta Crystallogr. F 75, 561-569 (2019).

10. Singal, B. et al. Crystallographic and solution structure of the N-terminal domain of the Rel protein from Mycobacterium tuberculosis. FEBS Lett. 591, 2323-2337 (2017).

11. Brown, A., Fernandez, I. S., Gordiyenko, Y. \& Ramakrishnan, V. Ribosomedependent activation of stringent control. Nature 534, 277-280 (2016).

12. Arenz, S. et al. The stringent factor RelA adopts an open conformation on the ribosome to stimulate ppGpp synthesis. Nucleic Acids Res. 44, 6471-6481 (2016).

13. Loveland, A. B. et al. Ribosome* RelA structures reveal the mechanism of stringent response activation. eLife 5, e17029 (2016).

14. Sun, D. et al. A metazoan ortholog of SpoT hydrolyzes ppGpp and functions in starvation responses. Nat. Struct. Mol. Biol. 17, 1188-1194 (2010).

15. Aravind, L. \& Koonin, E. V. The HD domain defines a new superfamily of metal-dependent phosphohydrolases. Trends Biochem. Sci. 23, 469-472 (1998).

16. Manav, M. C. et al. Structural basis for (p)ppGpp synthesis by the Staphylococcus aureus small alarmone synthetase RelP. J. Biol. Chem. 293, 3254-3264 (2018).

17. Steinchen, W. et al. Structural and mechanistic divergence of the small (p) ppGpp synthetases RelP and RelQ. Sci. Rep. 8, 2195 (2018).

18. Takada, H. et al. Ribosome association primes the stringent factor Rel for recruitment of deacylated tRNA to ribosomal A-site. Preprint at bioRxiv https://doi.org/10.1101/2020.01.17.910273 (2020).

19. Takada, H. et al. The C-terminal RRM/ACT domain is crucial for fine-tuning the activation of 'Long' RelA-SpoT homolog enzymes by ribosomal complexes. Front. Microbiol. 11, 277-307 (2020).

20. Steinchen, W. et al. Catalytic mechanism and allosteric regulation of an oligomeric (p)ppGpp synthetase by an alarmone. Proc. Natl Acad. Sci. USA 112, 13348-13353 (2015).

21. Okar, D. A. et al. PFK-2/FBPase-2: maker and breaker of the essential biofactor fructose-2,6-bisphosphate. Trends Biochem. Sci. 26, 30-35 (2001).

22. Gratani, F. L. et al. Regulation of the opposing (p)ppGpp synthetase and hydrolase activities in a bifunctional RelA/SpoT homologue from Staphylococcus aureus. PLoS Genet. 14, e1007514 (2018).

23. Ronneau, S. et al. Regulation of (p)ppGpp hydrolysis by a conserved archetypal regulatory domain. Nucleic Acids Res. 47, 843-854 (2019).

Publisher's note Springer Nature remains neutral with regard to jurisdictional claims in published maps and institutional affiliations.

(c) The Author(s), under exclusive licence to Springer Nature America, Inc. 2020 


\section{Methods}

Plasmids construction. The chemically synthesized gene of the $\operatorname{Rel}_{T t}$ protein from T. thermophilus (GenScript) was cleaved with XbaI and XhoI restriction enzymes (NEB) and transferred to pET21b (Invitrogene), which was cut with the same restriction enzymes and phosphorylated with alkaline phosphatase (NEB). The sequence of the resulting plasmid, pET21b-HisTEV-ttRel, was verified by sequencing (Eurofins Genomics). To produce a plasmid for the overexpression of the catalytic (N-terminal) domains of the $\mathrm{Rel}_{T t}$ protein $\left(\mathrm{Rel}_{T t}{ }_{T t}^{\mathrm{NTD}}\right)$, the C-terminal part of the gene (from the residue V356) was removed by amplifying the entire plasmid with primers (Sigma Aldrich) flanking the sequence to be deleted (Supplementary Table 3) with high-fidelity Q5 polymerase (Sigma Aldrich). The acquired PCR reaction mixtures were treated with DpnI (NEB) to remove the template plasmid. Finally, the PCR products were purified with a PCR purification column (Sigma), phosphorylated with polynucleotide kinase (Sigma) and ligated. The ligation mixture was transferred to E. coli MC1061 by electroporation. The resulting plasmid pET21b-HisTEV-ttRelcd (residues 1-355) was sequenced (Eurofins Genomics).

The point mutations were introduced into the $\mathrm{rel}_{T t}\left(\mathrm{rel}_{T t}^{N T D}\right)$ gene in pET21b-HisTEV-ttRel by amplifying the entire plasmid with high-fidelity Q5 polymerase (Sigma Aldrich) using primer pairs (Sigma Aldrich) where one primer carries the desired mutation in its $5^{\prime}$ end (Supplementary Table 3 ). The acquired PCR reactions mixtures were treated the same way as when constructing the C-terminal deletion of the enzyme. The removal and insertion of cysteine residues was carried out stepwise in the same way as described for single mutations, using the plasmid acquired in the previous step as the template for the next one.

To facilitate the cloning of the hydrolysis active site mutants, the ligation mixtures of $\operatorname{Rel}_{T t}(\mathrm{R} 43 \mathrm{~A}), \operatorname{Rel}_{T t}(\mathrm{D} 81 \mathrm{~N} / \mathrm{E}), \operatorname{Rel}_{T t}(\mathrm{R} 147 \mathrm{G}), \operatorname{Rel}_{T t}(\mathrm{Y} 49 \mathrm{~F})$ and $\operatorname{Rel}_{T t}(\mathrm{~N} 150 \mathrm{~A})$ were transformed to E. coli MC1061 already harboring the plasmid pBAD33-Mesh1. To obtain this plasmid, the chemically synthesized MESH1 gene of Homo sapiens (GenScript) was amplified with high-fidelity Q5 polymerase using primers Sac-RBS-Mesh-Fw and Mesh-Xho-Hind-Rev (Supplementary Table 3). The PCR product was cleaved using restriction enzymes HindIII and SacI (NEB) and transferred to the plasmid pBAD33 that was previously cleaved with the same enzymes and phosphorylated with alkaline phosphatase (NEB). The ligation mixture was transformed to E. coli MC1061 and verified by sequencing (Eurofins Genomics).

Protein expression and purification. HisTEV-Rel $\mathrm{R}_{T}$, its catalytic domain (HisTEV-Rel ${ }_{T t}{ }_{T t}^{\mathrm{NT}}$ ) or its point mutations (HisTEV-Rel ${ }_{T t}^{*}$ ) were expressed from the plasmids pET21b-HisTEV-Rel $\left.{ }_{T t}{ }^{*}\right)$ or pET21b-HisTEV-Rel ${ }_{T t}{ }^{\text {NTD }}$, respectively. The plasmids were transferred to E. coli BL21(DE3) by electroporation. The cells carrying the plasmid were grown to an optical density $\left(\mathrm{OD}_{600}\right)$ of 0.6 at $37^{\circ} \mathrm{C}$ in $\mathrm{LB}$ medium supplemented with ampicillin $\left(100 \mathrm{mgl}^{-1}\right)$, before induction of protein expression by addition of isopropyl- $\beta$-D-thiogalactoside (final concentration $0.5 \mathrm{mM}$ ) at $28^{\circ} \mathrm{C}$. The expression lasted for $5 \mathrm{~h}$ for $\operatorname{Rel}_{T t}{ }^{\mathrm{NTD}}$, or overnight for $\operatorname{Rel}_{T t}$.

For the expression of the HisTEV-Rel ${ }_{T t}^{*}$ with point mutations in the hydrolysis active centers (R43, Y49, D81, D146, R147 and N150) the pET21b-HisTEV-Rel ${ }_{T t}^{*}$ plasmids were transferred to $E$. coli BL21(DE3) cells that were already harboring the plasmid pBAD33-Mesh1. The proteins were expressed as previously described, but besides ampicillin the growth media was supplemented also with chloramphenicol $\left(10 \mathrm{mgl}^{-1}\right)$ and L-arabinose $(0.1 \%)$ to keep the Meshl expressed throughout the expression of the HisTEV-Rel ${ }_{T t}{ }^{\text {NTD }}$ hydrolysis mutant proteins. Next, the cells were collected and resuspended in resuspension buffer (50 mM Tris $\mathrm{pH} 8,1.5 \mathrm{M} \mathrm{KCl}, 2 \mathrm{mM} \mathrm{MgCl}, 1 \mathrm{mM}$ TCEP) supplemented with cOmplete protease inhibitor cocktail (Roche). If not used immediately for downstream applications, the cells were flash frozen in liquid nitrogen and stored at $-80^{\circ} \mathrm{C}$. In every case, cell disruption was performed using a cell cracker equilibrated with lysis buffer ( $50 \mathrm{mM}$ Tris $\mathrm{pH} 8,500 \mathrm{mM} \mathrm{NaCl}, 500 \mathrm{mM} \mathrm{KCl}, 1 \mathrm{mM}$ TCEP) supplemented with complete protease inhibitor cocktail (Roche). The pellet was separated from the supernatant by centrifugation for $30 \mathrm{~min}$ at $30,000 \mathrm{~g}$.

The supernatant was loaded onto a gravity flow TALON column, previously equilibrated with buffer A ( $25 \mathrm{mM}$ Tris $\mathrm{pH} 8,500 \mathrm{mM} \mathrm{NaCl}, 500 \mathrm{mM} \mathrm{KCl}, 10 \mathrm{mM}$ $\mathrm{MgCl}_{2}, 0.002 \%$ mellitic acid). The column was washed with 7 column volumes of buffer A and the bound protein eluted stepwise with buffers B1-3 (Buffer A containing 50, 125 and $500 \mathrm{mM}$ imidazole, respectively). The elution fractions containing His- $\mathrm{Re}_{T t}{ }_{T \text { ND }}$ were immediately concentrated using spin filters (Amicon) and loaded onto a GE Healthcare Superdex 200 16/60 gel filtration column equilibrated with GF buffer (50 mM Hepes pH 7.5, $500 \mathrm{mM} \mathrm{NaCl}, 500 \mathrm{mM} \mathrm{KCl}$, $10 \mathrm{mM} \mathrm{MgCl}_{2}, 0.002 \%$ mellitic acid; before crystallization procedures Tris $\mathrm{pH} 8$ was used as a buffering agent). The protein fractions were checked for purity with SDSPAGE. Pure protein was used immediately for further applications or stored in 35\% glycerol at $-20^{\circ} \mathrm{C}$ for up to 1 week.

Crystallization. Before crystallization, the His-tag of all protein variants was cleaved off by adding tobacco etch virus protease in a 1:200 molar ratio to the purified protein and incubating the mixture overnight at room temperature. The protein was analyzed on SDS gel for purity and cleavage. Additionally, the removal of the tag was confirmed by Western Blotting using an anti-polyHis antibody (Sigma Aldrich). To clear Rel $_{T t}^{\text {NTD }}$ from the His-tagged protease and free polyHis tag, the mixture was passed through a TALON column. The cleaved protein was subsequently concentrated for crystallization to $10-12 \mathrm{mg} \mathrm{ml}^{-1}$. The concentration of the protein solution was estimated using the theoretical extinction coefficient at $280 \mathrm{~nm}$ of the proteins as implemented in the ProtParam tool.

Crystallization conditions were screened at 20 and $4{ }^{\circ} \mathrm{C}$ by the sitting-drop vapor-diffusion method. The drops were set up in Swiss (MRC) 96-well two-drop UVP sitting-drop plates using the Mosquito HTS system (TTP Labtech). The drops consisting of $0.1 \mu \mathrm{l}$ protein and $0.1 \mu \mathrm{l}$ precipitant solution were equilibrated to $80 \mu \mathrm{l}$ precipitant solution in the reservoir. Crystallization conditions were tested with several commercially available screens: Crystal Screen I and II (Hampton Research), Helix, ProPlex, Pact Premier, JCSG, LMB and Morpheus II (Molecular Dimensions). For cocrystallization conditions of $\operatorname{Rel}_{T t}{ }_{\text {NTD }}$ with nucleotides, $\operatorname{Rel}_{T t}^{\text {NTD }}$ at a concentration of $10-12 \mathrm{mg} \mathrm{ml}^{-1}$ was mixed with $100 \mathrm{mM}$ of the nucleotides and incubated $10 \mathrm{~min}$ at room temperature. In every case, the nucleotides were also added to the suitable cryo-protecting solution used for harvesting the crystals and the samples were vitrified in liquid $\mathrm{N}_{2}$ for storage and transport before $\mathrm{X}$-ray exposure in the synchrotron.

X-ray diffraction data was collected at the SOLEIL synchrotron (Gif-sur-Yvette, Paris, France) on the Proxima 1 (PX1) and Proxima 2A (PX2A) beamlines using a PILATUS 6M detector and an Eiger detector, respectively. Because of the high anisotropic nature of the data from all the crystals we performed anisotropic cutoff and correction of the merged intensity data as implemented on the STARANISO server (http://staraniso.globalphasing.org/) using the DEBYE and STARANISO programs. In the case of the crystals of the $\operatorname{Rel}_{T t}{ }^{\text {NTD }}$-ppGpp-AMP complex, the analysis of the data suggested a resolution of $2.95 \AA$ (with $2.5 \AA$ in $a^{*}, 3.0 \AA$ in $b^{*}$ and $4.5 \AA$ in $c^{\star}$ ). In the case of the $\operatorname{Rel}_{T t}{ }^{\mathrm{NTD}}$-ppGp ${ }_{\mathrm{N}} \mathrm{p}$ complex, the analysis of the data suggested a resolution of $2.75 \AA$ (with $3.5 \AA$ in $a^{\star}, 3.5 \AA$ in $b^{\star}$ and $2.5 \AA$ in $c^{\star}$ ).

Structure determination. The data were processed with the XDS suite ${ }^{24}$ and scaled with XSCALE or Aimless. In all cases, the unit-cell content was estimated with the program MATTHEW COEF from the CCP4 program suite ${ }^{25}$. Molecular replacement was performed with Phaser ${ }^{26}$. The crystals of the $\operatorname{Rel}_{T t}{ }^{\mathrm{NTD}}$-ppGpp complex diffracted on average to $\sim 2.8 \AA$. We used the coordinates of $\operatorname{Rel}_{S e q}{ }^{\text {NTD }}$ as search model (PDB ID IVJ7) ${ }^{6}$. Because of the intrinsic interdomain dynamics of Rel catalytic domains we search for a molecular replacement (MR) solution using each catalytic domain as an independent ensemble. The MR solution from Phaser was used in combination with combined with Rosetta as implemented in the MR-Rosetta ${ }^{27}$ suit from the Phenix package ${ }^{28}$. MR-Rosetta could trace entirely the HD domain and 70\% of the SYN domain. After several iterations of manual building with $\operatorname{Coot}^{29}$ and maximum likelihood refinement as implemented in Buster/TNT ${ }^{30}$, the model was extended to cover all the residues $\left(R / R_{\text {free }}\right.$ of $17.9 / 22.9 \%)$.

In the case of the $\operatorname{Rel}_{T t}{ }^{\mathrm{NTD}}$-ppGpp-AMP complex, we used the coordinates of the individual HD and SYN domains from the structure of $\operatorname{Rel}_{T t}{ }^{\mathrm{NTD}}$-ppGpp as search model for MR in Phaser. The solution contained one molecule in the asymmetric unit. As with the $\operatorname{Rel}_{T t}{ }^{\mathrm{NTD}}$-ppGpp complex, we used MR-Rosetta ${ }^{27}$ after molecular replacement with Phaser. MR-Rosetta built the structure almost to completion and in the map resulting from MR-Rosetta, a clear density suggesting the presence of bound nucleotides in the SYN domain was observed. The structure was completed after several iterations of manual building with $\operatorname{Coot}^{29}$ and maximum likelihood refinement as implemented in Buster/ Buster/TNT ${ }^{30}$ to an $R / R_{\text {free }}$ of $20.9 / 24.4 \%$.

For the structure of $\operatorname{Rel}_{T t}{ }^{\mathrm{NTD}}$, we also used the coordinates of the individual HD and SYN domains from the structure of $\operatorname{Rel}_{T t}{ }^{{ }^{N T D}}$-ppGpp as search model for MR in Phaser. In this case, the analysis of the unit-cell content indicated the presence of three molecules in the asymmetric unit, which was confirmed after MR. We used MR-Rosetta ${ }^{27}$ after molecular replacement with Phaser for automated model reconstruction. The structure was completed after several iterations of manual building with $\operatorname{Coot}^{29}$ and maximum likelihood refinement as implemented in Buster/Buster/TNT ${ }^{30}$ to an $R / R_{\text {free }}$ of $22.1 / 27.4 \%$.

In every case, the geometrical restraints of all small molecules were generated with the Grade Web Server (http://grade.globalphasing.org). Supplementary Table 4 details all the X-ray data collection and refinement statistics.

ITC. All titrations were performed with an affinity ITC machine (TA Instruments) at $15^{\circ} \mathrm{C}$. Stock solutions of APCPP (Jena Biosciences) and GDP (Sigma Aldrich) of 650-670 $\mathrm{mM}$ were diluted in the protein buffer (50 mM HEPES pH 7.5; $500 \mathrm{mM} \mathrm{KCl} ; 500 \mathrm{mM} ; \mathrm{NaCl} ; 10 \mathrm{mM} \mathrm{MgCl}_{2} ; 1 \mathrm{mM}$ TCEP; $0.002 \%$ mellitic acid) to a final concentration $1.6-2.4 \mathrm{mM}$. The purified $\operatorname{Rel}_{T t}{ }^{\mathrm{NTD}}$ was concentrated by ultrafiltration (Amicon Ultra, $0.5 \mathrm{ml}, 30 \mathrm{kDa}$, Merck Millipore) to $80-100 \mu \mathrm{M}$. All final concentrations were verified by the absorption using a Nanodrop One (Thermo Scientific). All ITC measurements were performed by titrating $2 \mu \mathrm{l}$ of the nucleotide into the protein using a constant stirring rate of 75 r.p.m. All data were processed and analyzed using the NanoAnalyse and Origin software packages. Supplementary Fig. 10 shows the representative results for each titration and the thermodynamic parameters derived from the analysis of the ITC data are shown in Supplementary Table 2.

Sample preparation for smFRET. The $\operatorname{Rel}_{T t}{ }^{\mathrm{NTD}}$ variants $\left(\operatorname{Rel}_{T t}{ }^{\mathrm{NTD}}{ }_{6 / 287}\right.$ and $\operatorname{Rel}_{T t}^{\text {NTD }}{ }_{6 / 124}$ ) suitable for smFRET measurements were prepared in $50 \mathrm{mM}$ HEPES pH 7.5, $500 \mathrm{mM} \mathrm{KCl} ; 500 \mathrm{mM} \mathrm{NaCl} ; 10 \mathrm{mM} \mathrm{MgCl}_{2} ; 0.002 \%$ mellitic acid 
(FRET buffer) containing $1 \mathrm{mM}$ TCEP. The Alexa Fluor 647 (Thermo Fisher Scientific) and Atto 488 (ATTO-TEC) used as acceptor and donor were covalently attached to the protein via maleimide-Cys coupling. The concentrations of protein and dyes were determined by using a Nanodrop One (Thermo Scientific). Before labeling, TCEP was removed with a Sephadex G-25 PD10 desalting column (GE healthcare), which was equilibrated with FRET buffer. For the labeling procedure, the protein concentrated at $20-50 \mu \mathrm{M}$ was incubated in a $50-\mu \mathrm{l}$ reaction for $3 \mathrm{~h}$ at room temperature (or overnight at $4{ }^{\circ} \mathrm{C}$ ) with a 1.2-fold molar excess of each dye. After the labeling reaction, excess dye was removed with a Sephadex G-25 PD10 desalting column (GE healthcare), which was equilibrated with FRET buffer containing $1 \mathrm{mM}$ TCEP. Labeled protein was concentrated $(4-15 \mu \mathrm{M})$ with ultrafiltration (Amicon Ultra, $0.5 \mathrm{ml}, 30 \mathrm{kDa}$, Merck Millipore) and stored at $-20^{\circ} \mathrm{C}$ in $20 \%$ glycerol.

smFRET data recording. The stored protein was always diluted into FRET buffer before mixing with nucleotides in a reaction volume of $50 \mu \mathrm{l}$ containing labeled protein (at a final concentration of $300 \mathrm{pM}$ ). Nucleotides were always used at a final concentration of $500 \mathrm{nM}$ in the reaction volume. The bovine serum albumin-coated ( $1 \mathrm{mg} \mathrm{ml}^{-1}$ bovine serum albumin) coverslip (Nunc Lab-Tek Chambered Coverglass, Thermo Fisher Scientific) was rinsed three times with the FRET buffer $(30 \mu \mathrm{l})$, before depositing a 30 - $\mu$ l drop of the prepared proteinnucleotide mixture. Background (needed for calculating $E$ and $S$ parameters, and for lifetime and photon distribution analysis (PDA)) or scatter samples (needed for lifetime analysis) were prepared similarly but without protein or nucleotides (nucleotides had a negligible effect on the background).

Per sample, 1 -h datasets ( $10 \mathrm{~min}$ for background samples) were recorded at $22^{\circ} \mathrm{C}$ on a homebuilt multiparameter fluorescence detection microscope with pulsed interleaved excitation (MFD-PIE) as established ${ }^{31}$, with minor modifications. Emission from a pulsed 483-nm laser diode (LDH-P-C-470, PicoQuant) was cleaned up (Chroma ET485/20x, F49-482; AHF analysentechnik AG), emission from a 635-nm laser diode (LDH-P-C-635B, PicoQuant) was cleaned up (Chroma z635/10x, PicoQuant), and both lasers were alternated at $26.67 \mathrm{MHz}$ (PDL 828 Sepia II, PicoQuant), delayed $\sim 18 \mathrm{~ns}$ with respect to each other, and combined with a 483 -nm reflecting dichroic mirror in a single-mode optical fiber (coupler, 60FC-4-RGBV11-47; fiber, PMC-400Si-2.6-NA0123-APC-150-P, Schäfter + Kirchhoff). After collimation (60FC-L-4-RGBV11-47, $\mathrm{SuK})$, the linear polarization was cleaned up (CODIXX VIS-600- BC-W01, F22-601; AHF analysentechnik AG), and the light (100 mW of 483-nm light and $50 \mathrm{~mW}$ of $635-\mathrm{nm}$ light) was reflected into the back port of the microscope (IX70, Olympus) and upward (3-mm-thick full-reflective Ag mirror, F21-005 (AHF) mounted in a total internal reflection fluorescence filter cube for BX2/ IX2, F91-960; AHF analysentechnik AG) to the objective (UPLSAPO-60XW, Olympus). Sample emission was transmitted through a 3-mm-thick excitation polychroic mirror (Chroma zt470-488/640rpc, F58-PQ08; AHF analysentechnik AG), focused through a 75-mm pinhole (P75S, Thorlabs) with an achromatic lens (AC254-200-A-ML, Thorlabs), collimated again (AC254-50-A-ML, Thorlabs), and spectrally split (Chroma T560lpxr, F48-559; AHF analysentechnik AG). The blue range was filtered (Chroma ET525/50 m, F47-525, AHF analysentechnik AG), and polarization was split (PBS251, Thorlabs). The red range was also filtered (Chroma ET705/100 m, AHF analysentechnik AG), and polarization was split (PBS252, Thorlabs). Photons were detected on four avalanche photodiodes (PerkinElmer or EG\&G SPCM-AQR12/14, or Laser Components COUNT BLUE), which were connected to a time-correlated single-photon counting device (SPC-630, Becker \& Hickl) over a router (HRT-82, Becker \& Hickl) and power supply (DSN 102, PicoQuant). Signals were stored in 12-bit first-in-first-out files. Microscope alignment was carried out using fluorescence correlation spectroscopy on freely diffusing ATTO 488-CA and ATTO 655-CA (ATTO-TEC) and by connecting the detectors to a hardware correlator (ALV-5000/EPP) over a power splitter (PSM50/51, PicoQuant) for alignment by real-time fluorescence correlation spectroscopy. Instrument response functions were recorded one detector at-a-time in a solution of ATTO 488-CA or ATTO 655-CA in near-saturated centrifuged potassium iodide at a $25-\mathrm{kHz}$ average count rate for a total of $25 \times 10^{6}$ photons. Macrotime-dependent microtime shifting was corrected for two (blue/parallel and $\mathrm{red} /$ perpendicular) of four avalanche photodiodes using the Instrument response function data as input ${ }^{32}$.

SmFRET data analysis. Data was analyzed with the PAM software ${ }^{33}$ via standard procedures for MFD-PIE smFRET burst analysis ${ }^{34,35}$. Signals from each time-correlated single-photon count routing channel (corresponding to the individual detectors) were divided in time gates to discern 483-nm excited FRET photons from 635-nm excited acceptor photons. A two-color MFD all-photon burst search algorithm using a 500- $\mu$ s sliding time window (minimum of 50 photons per burst, minimum of five photons per time window) was used to identify single donor- and/or acceptor-labeled molecules in the fluorescence traces Double labeled single molecules were selected from the raw burst data using a kernel density estimator (ALEX-2CDE, $<15)^{36}$, that also excluded other artifacts. Bursts with a donor lifetime of $<0.01$ or $>5 \mathrm{~ns}$ ( $>3 \mathrm{~ns}$ for acceptor lifetimes) were omitted from the data. Sparse slow-diffusing aggregates were removed from the data by excluding bursts exhibiting a burst duration $>20 \mathrm{~ms}$. Data was corrected in this order to obtain the absolute stoichiometry parameter $S$ and absolute FRET efficiency $E$ : background subtraction, donor emission crosstalk correction, acceptor direct excitation correction and relative detection efficiency correction. The last value depends on the used dye combination, but also on the used detectors. By making histograms of $E$ versus measurement time, we corroborated that the distribution of $E$ was invariant over the duration of the measurement.

PDA. Static PDA was carried out to obtain the absolute interdye distance distribution as described before, assuming two (or more) Gaussian distributed states ${ }^{37}$. Practically, for each FRET data set, raw bursts were rebinned into 1-ms. Binned data was plotted in a raw (uncorrected) FRET efficiency $\left(E_{\mathrm{PR}}\right)$ versus uncorrected stoichiometry $\left(S_{\mathrm{PR}}\right)$ plot and only keeping the bins with $0.3<S_{\mathrm{PR}}<0.5$ efficiently removed burst sections containing complex acceptor photophysics or photobleaching. Furthermore, only bins with at least $10-20$ and maximally 250 photons (to reduce calculation time) were used for PDA analysis. A two- or three-state model for a Gaussian distance distribution was used to generate a library of simulated $E_{\mathrm{PR}}$ values, which was subsequently fitted to the experimental $E_{\mathrm{PR}}$ histogram using a reduced $\chi^{2}$-guided simplex search algorithm to obtain the amplitude, mean distance $R$ and width $\sigma$ of all Gaussian distributed substates ${ }^{38}$. A probability density function (PDF) was calculated per state using the $R$ and $\sigma$ parameters obtained from PDA analysis that describe the underlying Gaussian distributed states. The summed PDF was scaled to a total area of unity, with the PDF area of each state scaled to the corresponding fraction of molecules. Criteria for a good fit were a low reduced $\chi^{2}$ value, as well as a weighted-residual plot free of trends.

Preparation of $T$. thermophilus $70 \mathrm{~S}$ ribosomes. The $70 \mathrm{~S}$ ribosomes were prepared from T. thermophilus strain HB8 as described ${ }^{39}$ with minor modifications. Approximately $20 \mathrm{~g}$ of frozen $T$. thermophilus cell paste was resuspended in $50 \mathrm{ml}$ of buffer A $\left(100 \mathrm{mM} \mathrm{NH}_{4} \mathrm{Cl}, 10.5 \mathrm{mM} \mathrm{Mg}\right.$-acetate, $0.5 \mathrm{mM}$ EDTA, $6 \mathrm{mM}$ $\beta$-mercaptoethanol, $20 \mathrm{mM}$ Tris: $\mathrm{HCl} \mathrm{pH} \mathrm{7.5),} \beta$-mercaptoethanol (which should be added directly before use) supplemented with $1 \mathrm{mU}$ Turbo DNase (Thermo Fisher Scientific), 0.1 mM PMSF and opened by three passages on a high-pressure cell disrupter (Stansted Fluid Power) at $220 \mathrm{MPa}$. Lysed cells were clarified by centrifugation for $60 \mathrm{~min}$ at 30,000 r.p.m. (Ti 45 rotor, Beckman). The supernatant was removed, preventing the pellet from mixing with the cell debris and then carefully applied on sucrose cushion $\left(1.1 \mathrm{M}\right.$ sucrose, $0.5 \mathrm{M} \mathrm{NH}_{4} \mathrm{Cl}, 15 \mathrm{mM}$ Mg-acetate, $0.5 \mathrm{mM}$ EDTA, $3 \mathrm{mM} \beta$-mercaptoethanol, $20 \mathrm{mM}$ Tris: $\mathrm{HCl} \mathrm{pH} 7.5$ ) by loading $15 \mathrm{ml}$ of supernatant on the top of $40 \mathrm{ml}$ of sucrose cushion in $45 \mathrm{Ti}$ centrifuge tubes. After centrifugation for $8 \mathrm{~h}$ at 35,000 r.p.m. (Ti 45 rotor, Beckman) the ribosomes were pelleted and the supernatant was removed. Ribosomal pellets were resuspended in the buffer $\mathrm{A}$ and then applied to the sucrose cushions (45Ti centrifuge tubes), and centrifuged for $18 \mathrm{~h}$ at 28,000 r.p.m. (Ti 45 rotor, Beckman). Resulting pellets were resuspended in the buffer $\mathrm{A}$ and resolved on a $10-40 \%$ sucrose gradient in overlay buffer $\left(60 \mathrm{mM} \mathrm{NH}_{4} \mathrm{Cl}, 15 \mathrm{mM} \mathrm{Mg}(\mathrm{OAc})_{2}, 0.25 \mathrm{mM}\right.$ EDTA, $3 \mathrm{mM} \beta$-mercaptoethanol, $20 \mathrm{mM}$ Tris:HCl pH 7.5) in a zonal rotor Ti 15 (Beckman) (17 h at 21,000 r.p.m.). The peak containing pure $70 \mathrm{~S}$ ribosomes was pelleted by centrifugation ( $20 \mathrm{~h}$ at 35,000 r.p.m.), and the final ribosomal preparation was dissolved in HEPES:polymix buffer ${ }^{40}(20 \mathrm{mM}$ HEPES:KOH pH 7.5, 2 mM DTT, $5 \mathrm{mM} \mathrm{Mg}(\mathrm{OAc})_{2}, 95 \mathrm{mM} \mathrm{KCl}, 5 \mathrm{mM} \mathrm{NH}_{4} \mathrm{Cl}, 0.5 \mathrm{mM} \mathrm{CaCl}_{2}, 8 \mathrm{mM}$ putrescine, $1 \mathrm{mM}$ spermidine. The $70 \mathrm{~S}$ concentration was measured spectrophotometrically $\left(1 \mathrm{OD}_{260}\right.$ corresponds to $23 \mathrm{nM}$ of $70 \mathrm{~S}$ ) and ribosomes were aliquoted (50-100 $\mu \mathrm{l}$ per aliquot), snap frozen in liquid nitrogen and stored at $-80^{\circ} \mathrm{C}$.

Preparation of T. thermophilus 70S initiation complexes (70S IC). Initiation complexes were prepared by as established ${ }^{41}$, with minor modifications. The reaction mix containing T. thermophilus $70 \mathrm{~S}$ ribosomes (final concentration of $1 \mu \mathrm{M}$ ) with $1 \mu \mathrm{M} \mathrm{E}$. coli IF1, $1 \mu \mathrm{M}$ IF2, $1 \mu \mathrm{M}$ IF3, $1.5 \mu \mathrm{M}{ }^{3} \mathrm{H}$-fMet-tRNA ${ }_{\mathrm{i}}^{\text {fMet }}, 1.5 \mu \mathrm{M}$ messenger RNA MVF-Stop ( $5^{\prime}-$ GGCAAGGAGGAGAUAAGAAUGGUUUUCUAAUA-3'), 1 mM GTP and $2 \mathrm{mM}$ DTT in HEPES:Polymix buffer with $5 \mathrm{mM} \mathrm{Mg}(\mathrm{OAc})_{2}$ was incubated at $37^{\circ} \mathrm{C}$ for $30 \mathrm{~min}$. Then the ribosomes were pelleted through a sucrose cushion (1.1 M sucrose in HEPES:polymix buffer with $15 \mathrm{mM} \mathrm{Mg}^{2+}$ ) at 50,000 r.p.m. for $2 \mathrm{~h}$ (TLS-55, Beckman), the pellet was dissolved in HEPES:polymix buffer ( $5 \mathrm{mM}$ $\left.\mathrm{Mg}(\mathrm{OAc})_{2}\right)$, aliquoted, flash frozen in liquid nitrogen and stored at $-80^{\circ} \mathrm{C}$.

Biochemical assays. For $\mathrm{H}^{3}$-ppGpp synthesis assays, experiments were performed as described for $E$. coli ${ }^{42}$ with minor modifications. The reaction mixtures typically contained $120 \mathrm{nM}$ T. thermophilus $70 \mathrm{~S}$ IC(MV), $30 \mathrm{nM}$ Rel, guanosine nucleoside substrate $\left(300 \mu \mathrm{M} \mathrm{H}^{3}-\mathrm{GDP}\right)$, (ParkinElmer) E. coli $\mathrm{tRNA}^{\mathrm{Val}}$, all in HEPES:polymix buffer at $5 \mathrm{mM} \mathrm{Mg}^{2+}$ final concentration. After preincubation at $40^{\circ} \mathrm{C}$ for $3 \mathrm{~min}$, the reaction was started by the addition of prewarmed ATP to the final concentration of $1 \mathrm{mM}$, and $5 \mu \mathrm{l}$ aliquots were taken throughout the time course of the reaction and quenched with $4 \mu \mathrm{l} 70 \%$ formic acid supplemented with a cold nucleotide standard ( $4 \mathrm{mM}$ GDP and $4 \mathrm{mM} \mathrm{GTP}$ ) for ultraviolet (UV) shadowing. Individual quenched timepoints were spotted PEI-TLC plates (Macherey-Nagel) and nucleotides were resolved in $0.5 \mathrm{KH}_{2} \mathrm{PO}_{4} \mathrm{pH} 3.5$ buffer. The thin-layer chromatography plates were dried, cut into sections as guided by UV shadowing and ${ }^{3} \mathrm{H}$ radioactivity was quantified by scintillation counting in an Ecolite $(+)$ liquid scintillation cocktail (MP Biochemicals). 
For $\mathrm{H}^{3}$-ppGpp hydrolysis assays, the reaction mixtures typically contained $100 \mathrm{nM} \mathrm{Rel}_{T,}$, guanosine nucleoside substrate $\left(500 \mu \mathrm{M} \mathrm{H}^{3}\right.$-ppGpp), $0.5 \mathrm{mM}$ $\mathrm{MnCl}_{2}$, all in HEPES:Polymix buffer at $5 \mathrm{mM} \mathrm{Mg}^{2+}$ final concentration. After preincubation at $40^{\circ} \mathrm{C}$ for $3 \mathrm{~min}$, the reaction was started by the addition of prewarmed Rel and $5 \mu$ laliquots were taken throughout the time course of the reaction and quenched with $4 \mu \mathrm{l} 70 \%$ formic acid supplemented with a cold nucleotide standard (4 mM GDP) for UV shadowing.

Biochemical data analysis. To calculate the enzyme turnover, the individual kinetic data points in the form of substrate to product conversion ${ }^{3} \mathrm{H}-\mathrm{ppGpp}$ $\mathrm{CPM} /{ }^{3} \mathrm{H}-\mathrm{GDP} \mathrm{CPM}+{ }^{3} \mathrm{H}-\mathrm{ppGpp} \mathrm{CPM}$ (CPM refers to counts per million) for the synthesis reaction, and ${ }^{3} \mathrm{H}-\mathrm{GDP} C \mathrm{CPM} /{ }^{3} \mathrm{H}-\mathrm{ppGpp} \mathrm{CPM}+{ }^{3} \mathrm{H}$-GDP CPM for the hydrolysis reaction; were plotted as a function of time (hydrolysis reaction: 0 , 10,20 and $30 \mathrm{~min}$; synthesis reaction in the case of Rel tested either alone or in the presence of 70S IC: $0,10,20$ and 30 min; synthesis reaction in the case of Rel supplemented with 70S IC and deacylated tRNA ${ }^{\mathrm{Val}}:$ 0, 5, 10 and $15 \mathrm{~min}$ ).

From these graphs, the turnovers were estimated the slope of the line fit (conversion $=$ turnover $\times$ time + background) to the kinetic data with a using Igor Pro (Wave Metrics, Inc.). The error bars represent standard deviations of the estimates.

Reporting Summary. Further information on research design is available in the Nature Research Reporting Summary linked to this article.

\section{Data availability}

All the structures have been deposited in the PDB database with the following accession numbers; $6 \mathrm{~S} 2 \mathrm{~V}, 6 \mathrm{~S} 2 \mathrm{~T}$ and $6 \mathrm{~S} 2 \mathrm{U}$. All data needed to evaluate the conclusions in the paper are present in the paper and/or the Methods. Additional data related to this paper may be requested from the authors.

\section{References}

24. Kabsch, W. XDS. Acta Crystallogr D 66, 125-132 (2010).

25. Collaborative Computational Project, N. The CCP4 suite: programs for protein crystallography. Acta Crystallogr. D 50, 760-763 (1994).

26. McCoy, A. J. et al. Phaser crystallographic software. J. Appl. Crystallogr. 40, 658-674 (2007).

27. Terwilliger, T. C. et al. phenix.mr_rosetta: molecular replacement and model rebuilding with Phenix and Rosetta. J. Struct. Funct. Genomics 13, 81-90 (2012).

28. Afonine, P. V. et al. Towards automated crystallographic structure refinement with phenix.refine. Acta Crystallogr. D 68, 352-367 (2012).

29. Emsley, P. \& Cowtan, K. Coot: model-building tools for molecular graphics. Acta Crystallogr. D 60, 2126-2132 (2004).

30. Smart, O. S. et al. Exploiting structure similarity in refinement: automated NCS and target-structure restraints in BUSTER. Acta Crystallogr. D 68, 368-380 (2012).

31. Talavera, A. et al. Phosphorylation decelerates conformational dynamics in bacterial translation elongation factors. Sci. Adv. 4, eaap9714 (2018).

32. Otosu, T., Ishii, K. \& Tahara, T. Note: simple calibration of the counting-rate dependence of the timing shift of single photon avalanche diodes by photon interval analysis. Rev. Sci. Instrum. 84, 036105 (2013).

33. Schrimpf, W., Barth, A., Hendrix, J. \& Lamb, D. C. PAM: a framework for integrated analysis of imaging, single-molecule, and ensemble fluorescence data. Biophys. J. 114, 1518-1528 (2018)

34. Hellenkamp, B. et al. Precision and accuracy of single-molecule FRET measurements-a multi-laboratory benchmark study. Nat. Methods 15, 669 (2018)

35. Kudryavtsev, V. et al. Combining MFD and PIE for accurate single-pair Forster resonance energy transfer measurements. Chem. Phys. Chem. 13, 1060-1078 (2012).
36. Tomov, T. E. et al. Disentangling subpopulations in single-molecule FRET and ALEX experiments with photon distribution analysis. Biophys. J. 102, 1163-1173 (2012)

37. Antonik, M., Felekyan, S., Gaiduk, A. \& Seidel, C. A. Separating structural heterogeneities from stochastic variations in fluorescence resonance energy transfer distributions via photon distribution analysis. J. Phys. Chem. B 110, 6970-6978 (2006).

38. Kalinin, S. et al. A toolkit and benchmark study for FRET-restrained high-precision structural modeling. Nat. Methods 9, 1218-1225 (2012).

39. Polikanov, Y. S., Steitz, T. A. \& Innis, C. A. A proton wire to couple aminoacyl-tRNA accommodation and peptide-bond formation on the ribosome. Nat. Struct. Mol. Biol. 21, 787-793 (2014).

40. Antoun, A., Pavlov, M. Y., Tenson, T. \& Ehrenberg, M. M. Ribosome formation from subunits studied by stopped-flow and Rayleigh light scattering. Biol. Proced. Online 6, 35-54 (2004).

41. Murina, V., Kasari, M., Hauryliuk, V. \& Atkinson, G. C. Antibiotic resistance $\mathrm{ABCF}$ proteins reset the peptidyl transferase centre of the ribosome to counter translational arrest. Nucleic Acids Res. 46, 3753-3763 (2018).

42. Kudrin, P. et al. The ribosomal A-site finger is crucial for binding and activation of the stringent factor RelA. Nucleic Acids Res. 46, 1973-1983 (2018).

\section{Acknowledgements}

We acknowledge the use of the synchrotron-radiation facility at the SOLEIL synchrotron Gif-sur-Yvette, France, under proposals 20150717, 20160750 and 20170756. We also thank the staff from Swing, PROXIMA-1 and PROXIMA-2A beamlines at SOLEIL for assistance with data collection. This work was supported by grants from the Fonds National de Recherche Scientifique, nos. FNRS-EQP U.N043.17F, FRFS-WELBIO CR-2017S-03 and FNRS-PDR T.0066.18, and the Joint Programming Initiative on Antimicrobial Resistance (grant no. JPI-EC-AMR-R.8004.18-) to A.G.-P. The Program 'Actions de Recherche Concertée' 2016-2021 and Fonds d'Encouragement à la Recherche from the ULB, Fonds Jean Brachet and the Fondation Van Buren to A.G.-P.; the Molecular Infection Medicine Sweden, Swedish Research council (grant no. 2017-03783), and Ragnar Söderberg foundation fellowship to V.H.; J. Hendrix and J. Hofkens are grateful to the Research Foundation Flanders (FWO Vlaanderen, grant no. G0B4915N) and large infrastructure grant (no. ZW15_09 GOH6316N) and the KU Leuven Research Fund (no. C14/16/053); J.Hofkens thanks financial support of the Flemish government through long-term structural funding Methusalem (CASAS2, Meth/15/04). K.V.N. was supported by a PhD grant from the Fonds National de Recherche Scientifique FNRS-FRIA. N.V. acknowledges the Agency for Innovation by Science and Technology in Flanders for a PhD grant. H. Tamman was supported by a Chargé de Recherches fellowship from the FNRS (no. CR/DM-392). H. Takada was supported by the postdoctoral grant from the Umeå Centre for Microbial Research (UCMR)

\section{Author contributions}

H. Tamman, K.V.N., N.V., D.S. and A.T. performed biophysical, structural biology and smFRET experiments. H. Takada performed biochemical assays. Y.P. was involved in the initial steps of the preparation of T. thermophilus ribosomes. J. Hendrix and J. Hofkens supervised the smFRET data analysis. V.H., J. Hendrix and A.G.-P. designed research and wrote the paper.

\section{Competing interests}

The authors declare no competing interests.

\section{Additional information}

Supplementary information is available for this paper at https://doi.org/10.1038/ s41589-020-0520-2.

Correspondence and requests for materials should be addressed to V.H., J.H. or A.G.-P.

Reprints and permissions information is available at www.nature.com/reprints. 


\section{Reporting Summary}

Nature Research wishes to improve the reproducibility of the work that we publish. This form provides structure for consistency and transparency in reporting. For further information on Nature Research policies, see Authors \& Referees and the Editorial Policy Checklist.

\section{Statistics}

For all statistical analyses, confirm that the following items are present in the figure legend, table legend, main text, or Methods section.

$\mathrm{n} / \mathrm{a}$ Confirmed

$\bigotimes$ The exact sample size $(n)$ for each experimental group/condition, given as a discrete number and unit of measurement

$\square$ \A statement on whether measurements were taken from distinct samples or whether the same sample was measured repeatedly

\ The statistical test(s) used AND whether they are one- or two-sided

$\triangle$ Only common tests should be described solely by name; describe more complex techniques in the Methods section.

Х $\square$ A description of all covariates tested

Х $\square$ A description of any assumptions or corrections, such as tests of normality and adjustment for multiple comparisons

A full description of the statistical parameters including central tendency (e.g. means) or other basic estimates (e.g. regression coefficient) AND variation (e.g. standard deviation) or associated estimates of uncertainty (e.g. confidence intervals)

$\square$ For null hypothesis testing, the test statistic (e.g. $F, t, r$ ) with confidence intervals, effect sizes, degrees of freedom and $P$ value noted Give $P$ values as exact values whenever suitable.

Х $\square$ For Bayesian analysis, information on the choice of priors and Markov chain Monte Carlo settings

Х $\square$ For hierarchical and complex designs, identification of the appropriate level for tests and full reporting of outcomes

$\triangle \square$ Estimates of effect sizes (e.g. Cohen's $d$, Pearson's $r$ ), indicating how they were calculated

Our web collection on statistics for biologists contains articles on many of the points above.

\section{Software and code}

Policy information about availability of computer code

Data collection

Data analysis
We did not generate any new software code for our work.

All the softwares used for the analysis of our data are described in the Material and Methods of the paper. They consists of Microcal Origin and NanoAnalyze for the analysis of all the calorimetric data. The crystallographic data were analyzed with the XDS, CCP4, PHENIX autoPROC and BUSTER/TnT suites. The SmFRET data were analyzed with the PAM software package implemented in MatLab which was also used for the PCA of the data.

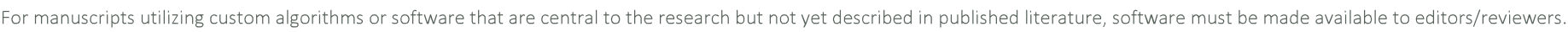
We strongly encourage code deposition in a community repository (e.g. GitHub). See the Nature Research guidelines for submitting code \& software for further information.

\section{Data}

Policy information about availability of data

All manuscripts must include a data availability statement. This statement should provide the following information, where applicable:

- Accession codes, unique identifiers, or web links for publicly available datasets

- A list of figures that have associated raw data

- A description of any restrictions on data availability

We described in our paper 3 crystal structures. The coordinates of these structures have been deposited in the PDB under the accession codes: 6S2V, 6S2T, 6S2U. 
Please select the one below that is the best fit for your research. If you are not sure, read the appropriate sections before making your selection.

Х Life sciences

Behavioural \& social sciences

Ecological, evolutionary \& environmental sciences

For a reference copy of the document with all sections, see nature.com/documents/nr-reporting-summary-flat.pdf

\section{Life sciences study design}

All studies must disclose on these points even when the disclosure is negative.

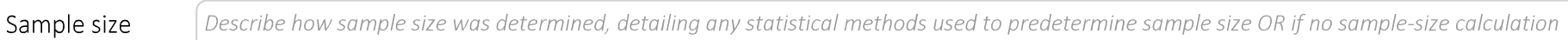
was performed, describe how sample sizes were chosen and provide a rationale for why these sample sizes are sufficient.

Data exclusions Describe any data exclusions. If no data were excluded from the analyses, state so OR if data were excluded, describe the exclusions and the rationale behind them, indicating whether exclusion criteria were pre-established.

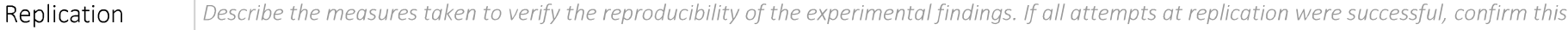
OR if there are any findings that were not replicated or cannot be reproduced, note this and describe why.

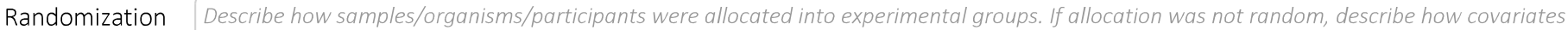
were controlled OR if this is not relevant to your study, explain why.

Blinding Describe whether the investigators were blinded to group allocation during data collection and/or analysis. If blinding was not possible, describe why OR explain why blinding was not relevant to your study.

\section{Reporting for specific materials, systems and methods}

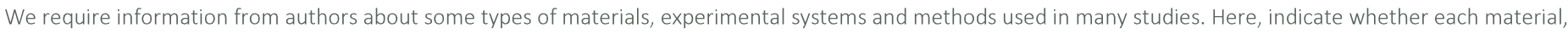

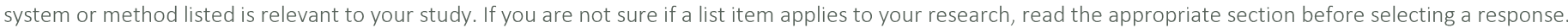

Materials \& experimental systems

n/a Involved in the study

叉 $\square$ Antibodies

Х $\square$ Eukaryotic cell lines

Methods

\ $\square$ Palaeontology $\mathrm{n} / \mathrm{a}$ Involved in the study

Х $\square$ ChIP-seq

X $\square$ Animals and other organisms

Х $\square$ Flow cytometry

X $\square$ Human research participants

Х $\square$ Clinical data 